Article

\title{
A Quantitative Analysis of an EEG Epileptic Record Based on Multiresolution Wavelet Coefficients
}

\author{
Mariel Rosenblatt ${ }^{1, *}$, Alejandra Figliola ${ }^{1}$, Gustavo Paccosi ${ }^{1}$, Eduardo Serrano $^{2}$ and \\ Osvaldo A. Rosso ${ }^{3,4}$
}

${ }^{1}$ Instituto del Desarrollo Humano, Universidad Nacional de General Sarmiento, Juan María Gutiérrez 1150, Provincia de Buenos Aires, Argentina;

E-Mails:afigliol@ungs.edu.ar (A.F.); gpaccosi@ungs.edu.ar (G.P.)

${ }^{2}$ Centro de Matemática Aplicada, Escuela de Ciencia y Tecnología, Universidad Nacional de San Martín, Irigoyen 3100, Provincia de Buenos Aires, Argentina; E-Mail:eduardo.eduser@gmail.com

${ }^{3}$ Instituto de Física, Universidade Federal de Alagoas, BR 104 Norte km 97, 57072-970 Maceió, Alagoas, Brazil; E-Mail: oarosso@gmail.com

${ }^{4}$ Instituto Tecnológico de Buenos Aires (ITBA), Av. Eduardo Madero 399 (C1106ACD),

Ciudad Autónoma de Buenos Aires, Argentina

* Author to whom correspondence should be addressed; E-Mail: mrosen@ungs.edu.ar;

Tel.:+54-11-4469-7708.

External Editor: Kevin H. Knuth

Received: 22 August 2014; in revised form: 7 November 2014 / Accepted: 11 November 2014 / Published: 17 November 2014

\begin{abstract}
The characterization of the dynamics associated with electroencephalogram (EEG) signal combining an orthogonal discrete wavelet transform analysis with quantifiers originated from information theory is reviewed. In addition, an extension of this methodology based on multiresolution quantities, called wavelet leaders, is presented. In particular, the temporal evolution of Shannon entropy and the statistical complexity evaluated with different sets of multiresolution wavelet coefficients are considered. Both methodologies are applied to the quantitative EEG time series analysis of a tonic-clonic epileptic seizure, and comparative results are presented. In particular, even when both methods describe the dynamical changes of the EEG time series, the one based on wavelet leaders presents a better time resolution.
\end{abstract}

Keywords: wavelet analysis; wavelet leaders; entropy; statistical complexity; local regularity; EEG 
MSC classifications: 65T60; 94A12; 26A16; 37M10; 28D20

\section{Introduction}

Human brain electrical activity can be measured from the scalp in a non-invasive way by means of electroencephalography. Due to the conductive properties of biological tissue, this activity reflects the effect of proximal and distal sources of synchronized neuronal activities [1]. The electroencephalogram (EEG) is one of the oldest techniques used in clinical neurophysiology to access information about the condition of the brain. Although EEG recordings have been in clinical use for more than half a century, conventional EEG analysis relies mostly on visual inspection and pattern recognition. Even when this methodology is quite useful, the visual inspection of the EEG is subjective and hardly allows any systematization [1]. In order to overcome this obstacle, quantitative EEG analysis (qEEG) introduces objective measures reflecting the characteristics of brain activity, as well as its associated dynamics.

The analysis of EEG signals always involves queries of quantification, i.e., the ability to state objective data in numerical and/or graphical form. Processing of information by the brain is reflected in dynamical changes of electrical activity in time, frequency and space. Therefore, the study of this process requires methods that can describe the variation of the signal in time and frequency. An attractive property of these quantifiers is that they are related to physical properties; so their interpretation and the implications of their results are straightforward. Thus, in order to understand the associated dynamics of the EEG time series, one can study the temporal evolution of these associated quantifiers and reach conclusions about their behavior under different pathologies and diseases.

In recent years, different research groups have reported very interesting results in the characterization of time evolution of epileptic EEG records (EEG time series provided by depth and cortical electrodes) using nonlinear dynamical invariant metrics quantifiers. In these studies, the EEG during epileptic seizure and the background EEG (pre- and post-seizure) were analyzed, following the temporal evolution of signal dimensionality (associated with the measurement of the correlation dimension, $D_{2}$ ) [2,3] and the chaoticness degree (through the largest Lyapunov exponent, $\Lambda_{\max }$ ) [4-7]. The main finding of these reports is a reduction of these two quantifiers during an epileptic seizure, suggesting that a transition takes place at the seizure onset in the dynamic behavior of the neural network, from a complex behavior to a simpler one [8]. Furthermore, some researchers report a significant decrease of these quantifiers a few minutes before the seizure onset, raising the hypothesis that epileptic seizure could be predicted $[3,5,6]$. However, despite the obvious physiological relevance of such findings, a basic requirement for nonlinear dynamic metric tools (chaos theory) to be applied to experimental data is the stationarity of the time series, which suggests that the time series is representative of a unique and stable attractor. Unfortunately, this is not the case with EEGs. To make the situation even worse, the evaluation of $D_{2}$ and $\Lambda_{\max }$ (defined as asymptotic properties of the attractor) requires the use of long time recordings.

Another group of qEEG methods are based on time-frequency analysis. A natural approach to quantify the degree of order of a complex signal is to consider its spectral entropy, as defined from the Fourier power spectrum [9]. The spectral entropy is a measure of how concentrated or wide-spread 
the Fourier power spectrum of a signal is. However, the Fourier transform (FT) requires stationarity of the signal, and EEGs are highly non-stationary. Furthermore, the FT does not yield the time evolution of the pertinent frequency patterns. Consequently, the spectral entropy does not get defined as a function of time.

The disadvantages of the spectral entropy defined from the FT can be partially overcome by using a short-time Fourier transform (STFT). Powell and Percival [9] have defined a time evolving entropy from the STFT by using a Hanning window. With this approach, the FT is applied to time-evolving windows of a few seconds of data refined with an appropriate function, so that the time-evolution of the frequencies can be followed. The stationarity requirement is partially satisfied by considering the signals as quasi-stationary for a few seconds. Due to the uncertainty principle [10-12], one critical limitation appears when windowing data: if the window is too narrow, the frequency resolution will be poor. Conversely, if the window is too wide, the time localization will be less precise.

All of the above-mentioned difficulties can be overcome by appealing to the wavelet transform [10-12], an efficient time-frequency decomposition method. In particular, the orthogonal discrete wavelet transform (ODWT) makes no assumptions about a record's stationarity (it does not need to reconstruct any attractor of the dynamical system), and it works only with its measurable response, that is the time series. Then, taking the ODWT of the EEG signal as a basic element, represented by the corresponding wavelet coefficients, the relative wavelet energy (RWE) can be evaluated. This provides information about the relative energy associated with the different frequency bands present in the EEG signal and their corresponding degree of importance. The RWE can be considered as the energy probability distribution, which, in turn, can be used to evaluate information theory quantifiers, such as Shannon entropy and statistical complexity measures, whose corresponding time evolution can be followed with an optimal time-frequency resolution [13-15]. This enables one to accurately characterize the evolution of the underlying brain dynamics that generate the corresponding EEG time series under analysis [15-25]. Moreover, this methodology based on the use of ODWT coefficients can be used to study and characterize brain electrical signals of a short duration, such as event-related potentials (ERPs) and evoked potentials (EPs) [26-31].

An alternative approach to the previous ODWT methodology has been proposed in [32]. In that work, we analyzed the dynamics of time series by defining a discrete probability distribution at each sample time, based on the computation of multiresolution quantities, called wavelet leaders [33] to evaluate the Shannon entropy. This way, information theory quantifiers can be defined at each sample time.

The wavelet leaders, computed in terms of the local suprema of wavelet coefficients, were introduced by S. Jaffard in [33], who finds a formula that yields the upper box dimension of a graph of a function. These multiresolution coefficients have proven to be a useful tool in signal and image processing. S. Jaffard reformulates the wavelet characterization of the local regularity of a function in terms of the wavelet leaders and also introduces a wavelet leader-based multifractal formalism in [34], using the wavelet leaders instead of using the wavelet coefficients or the continuous wavelet transform in the formula proposed in [35]. This wavelet leader-based multifractal formalism results in an effective and well-founded procedure to estimate the multifractal spectrum of different data signals and images [36-38] with a low computational cost. Recently, a wavelet leader oscillation formalism has also been proposed in order to analyze oscillating singularities in real signal data [39]. 
In this view, the pointwise wavelet leader entropy has been formulated in [32] as an alternative quantifier that refines the methodology based on ODWT coefficients. To evaluate the Shannon entropy, it uses a discrete probability distribution based on wavelet leaders at each time, instead of computing relative wavelet energy in non-overlapping temporal windows of a given size, limited by the length and the frequency content of the signal. In this work, we also evaluate the statistical complexity based on wavelet leaders, introducing the pointwise wavelet leader statistical complexity. In addition, these wavelet leader-based information quantifiers are correlated with the pointwise Hölder exponent, a temporal exponent that captures the regularity of the variation of a function, quantifying how rugous or spiky the graph of a function is. The pointwise Hölder exponent is the usual regularity exponent considered in signal and image processing, and it has been used for formulating the multifractal analysis for functions (for details, see [34] and the references therein).

The aim of the present work is to study an EEG signal corresponding to a tonic-clonic epileptic seizure avoiding noise-related limitations and using quantitative analysis based on multiresolution wavelet coefficients. In particular:

- We review the methodology based on orthogonal wavelet transform (ODWT) proposed by us in [13-15].

- We develop a novel extension of this methodology, using the wavelet leaders for the corresponding information theory quantifier evaluation.

- We exemplify both treatments with the analysis of a scalp epileptic EEG signal and compare the results of both methodologies for a scalp EEG recorded in the right central location (Channel C4), obtained from one patient.

We would like to emphasize the novelty of the use of wavelet leaders and the corresponding information theory-based quantifiers in the quantitative EEG analysis of epileptic records. In particular, even when results are shown for only one patient and one seizure, the new methodology based on wavelet leaders was applied to the complete dataset (patients and seizures) analyzed by us in a previous work [14]. Similar results to those described in the present work were found, confirming the applicability of this new methodology in the EEG analysis.

This article is organized in the following way. In Section 2, the wavelet methodology and information theory quantifiers are presented. This section includes both treatments, those based on orthogonal discrete wavelet transform and the one based on wavelet leaders, as well as the evaluation of the corresponding quantifiers. In Section 3, the clinical data and the experimental setup are given, the analysis of the epileptic EEG record (time series) with the two approaches are presented and results are discussed. Finally, in Section 4, a summary is given.

\section{Wavelet Methodology and Information Theory Quantifiers}

\subsection{Wavelet Transform}

The wavelet representation provides precise measurements regarding (a) when and to what degree transient events take place in a signal and (b) when and how the frequency contents of the signal change over time [10-12]. The wavelet is a simple and quickly vanishing oscillating function. Unlike the sine 
and cosine of Fourier analysis, which are precisely localized in frequency, but extend infinitely in time, wavelets are relatively localized in both time and frequency. Furthermore, wavelets are band-limited; they are composed of not one, but a relatively limited range of several frequencies.

A wavelet family $\left\{\psi_{a, b}\right\}$ is the set of elemental functions generated by (i) scaling and (ii) translation of an admissible mother wavelet $\psi(x)[11]$ :

$$
\psi_{a, b}(x)=a^{-1 / 2} \psi\left(\frac{x-b}{a}\right)
$$

where $a, b \in \mathbb{R}, a>0$ are the dilation (scale) and translation factors, respectively. Usually the variable $x$ is associated with time.

The continuous wavelet transform (CWT) of a signal $f(x) \in L^{2}(\mathbb{R})$ - the space of real square summable functions - is defined as the correlation between the function (signal) $f(x)$ with the family wavelet $\psi_{a, b}(x)$ for each $a$ and $b:[10-12]$ :

$$
W f(a, b)=a^{-1 / 2} \int_{-\infty}^{+\infty} f(x) \psi^{*}\left(\frac{x-b}{a}\right) d x=\left\langle f, \psi_{a, b}\right\rangle
$$

where $*$ means complex conjugation. This equation can be inverted in order to achieve a perfect reconstruction of the original signal. It measures the variation of $f(x)$ in a neighborhood of $b$ proportional to $a$, and $\left\langle f, \psi_{a, b}\right\rangle$ represent the wavelet coefficients. In principle, the CWT produces an infinite number of coefficients. The information displayed at closely-spaced scales or at closely-spaced time points is highly correlated. As a consequence, the CWT provides a redundant representation of the signal under analysis. It is also time consuming to compute directly.

\subsection{Discrete Wavelet Transform and Quantifiers}

The discrete wavelet transform (DWT) is defined by giving a non-redundant, highly efficient wavelet representation that can be implemented with a simple recursive filter scheme, and the original signal reconstruction can be obtained by an inverse filtering operation. For (i) a special election of the mother wavelet function $\psi_{a, b}$ and (ii) the discrete set of parameters, $a_{j}=2^{-j}$ and $b_{j, k}=2^{-j} k$ with $j, k \in \mathbb{Z}$ (the set of integers), the family $\mathcal{F}=\left\{\psi_{j, k}(x)=2^{j / 2} \psi\left(2^{j} x-k\right)\right\}$ constitutes an orthonormal basis of $L^{2}(\mathbb{R})$, the space of finite-energy signals. Each scale $a_{j}=2^{-j}$ is related to a given frequency band, and $j$ is known as the resolution level.

Then, the signal $f(x) \in L^{2}(\mathbb{R})$ can be recovered by:

$$
f(x)=\sum_{j \in \mathbb{Z}} \sum_{k \in \mathbb{Z}} C_{j, k} \psi_{j, k}(x)
$$

in the $L^{2}(\mathbb{R})$ sense, where the inner-product coefficients,

$$
C_{j, k}=\left\langle f, \psi_{j, k}\right\rangle=\int_{-\infty}^{+\infty} f(x) \psi_{j, k}(x) d x,
$$

are the wavelet coefficients.

The DWT produces only as many coefficients as there are samples within the signal under analysis $f(x)$, without the loss of any information at all. These wavelet coefficients provide full information 
in a simple way and a direct estimation of local energies at the different relevant scales. Moreover, the information can be organized in a multiresolution manner, yielding a hierarchical scheme of nested subspaces in $L^{2}(\mathbb{R})$. This scheme brought to light a link with filter banks and a fast wavelet transform algorithm decomposing signals of $N$ samples with $\mathcal{O}(N)$ operations. We refer the reader to [11,12,40,41] for detailed expositions of these topics.

Among several alternatives, we use a B-cubic spline function as a mother wavelet. It combines smoothness with numerical advantages in a suitable proportion (for a complete discussion, see Unser [42] and Thévenaz et al. [43]).

The signal is assumed to be given by the $N$ sampled values $\{f(n): n=1, \cdots, N\}$ corresponding to a uniform time grid with sampling time $\Delta t$. If the decomposition is carried out over all resolution levels $j=1$ to $j_{\text {max }} \leq \log _{2}(N)$, according to Equation (3), the wavelet expansion of the signal is,

$$
f(x) \cong \sum_{j=1}^{j_{\max }} \sum_{k} C_{j, k} \psi_{j, k}(x),
$$

where wavelet coefficients $C_{j, k}$ can be interpreted as the local residual errors between successive signal approximations at scales $j-1$ and $j$, respectively, and contain the information of the signal $f(x)$ corresponding to the frequency interval $2^{j-j_{\max }-2} \omega_{s} \leq|\omega| \leq 2^{j-j_{\max }-1} \omega_{s}$, where $\omega_{s}$ is the sample frequency.

Since the family $\mathcal{F}=\left\{\psi_{j, k}(x)\right\}$ is an orthonormal basis for $L^{2}(\mathbb{R})$, the concept of energy is linked with the usual notions derived from the Fourier theory. The energy at resolution level $j=1, \cdots, j_{\max }$ will be given by:

$$
E_{j}=\sum_{k}\left|C_{j, k}\right|^{2}
$$

The total energy, denoted by $E_{t o t}=\|f\|^{2}$ of the signal is:

$$
E_{t o t}=\sum_{j=1}^{j_{\max }} E_{j}=\sum_{j=1}^{j_{\max }} \sum_{k}\left|C_{j, k}\right|^{2} .
$$

Finally, we define the normalized $\rho_{j}$-values, which represent the relative wavelet energy (RWE):

$$
\rho_{j}=\frac{E_{j}}{E_{t o t}}
$$

for the resolution levels $j=1, \cdots, j_{\max }$.

The $\rho_{j}$ yield, at different scales, the probability distribution for the energy. Clearly, $\sum_{j=1}^{j_{\max }} \rho_{j}=1$. The distribution $\mathcal{P}^{(w)}=\left\{\rho_{j}: j=1, \cdots, j_{\text {max }}\right\}$ can be considered as a time-scale probability density that constitutes a suitable tool for detecting and characterizing specific phenomena in both the time and the frequency planes [13-15].

An information theory quantifier can be defined as a measure that is able to characterize some property of the probability distribution function (pdf) associated with an observable or measurable quantity. Entropy, regarded as a measure of uncertainty, is the most paradigmatic example [44,45]. Indeed, Kolmogorov and Sinai converted Shannon's information theory into a powerful tool for the study of dynamical systems [46,47]. 
The Shannon entropy [44] gives a useful criterion to analyze and compare probability distributions. It provides a measure of the information contained in any distribution. We define the Shannon wavelet entropy (SWS) [13-15] as:

$$
S\left[\mathcal{P}^{(w)}\right]=-\sum_{j=1}^{j_{\max }} \rho_{j} \log _{2}\left(\rho_{j}\right),
$$

where $\mathcal{P}^{(w)}$ denotes the probability energy wavelet distribution given by Equation (8).

Thus, the Shannon wavelet entropy appears as a measure of the degree of order/disorder of the signal, giving useful information about the underlying dynamical process associated with the signal [13-15]. Indeed, a very ordered process can be represented by a periodic mono-frequency signal (that is, a signal with a narrow band spectrum). A wavelet representation of such a signal will be resolved at one unique wavelet resolution level, i.e., all of the relative wavelet energy will be (almost) zero, except at the wavelet resolution level, which includes the representative signal frequency. For this special level, the relative wavelet energy will (in our chosen energy units) almost equal unity. As a consequence, the Shannon wavelet entropy will acquire a very small, vanishing value. A signal generated by a totally random process can be taken as representative of a very disordered behavior. This kind of signal will have a wavelet representation with significant contributions coming from all frequency bands. Moreover, one could expect that all contributions will be of the same order. Consequently, the relative wavelet energy will be almost equal at all resolution levels, and the Shannon wavelet entropy will acquire its maximum possible value. That is, in this case, the probability distribution will be represented by a uniform relative wavelet distribution given by $\mathcal{P}_{e}^{(w)}=\left\{\rho=1 / j_{\max }, \forall j=1, \cdots, j_{\max }\right\}$ and $S\left[\mathcal{P}_{e}^{(w)}\right]=S_{\text {max }}=\log _{2}\left(j_{\max }\right)$. We define the normalized Shannon wavelet entropy (normalized SWS) given by:

$$
H\left[\mathcal{P}^{(w)}\right]=\frac{S\left[\mathcal{P}^{(w)}\right]}{S\left[\mathcal{P}_{e}^{(w)}\right]}=\frac{S\left[\mathcal{P}^{(w)}\right]}{\log _{2}\left(j_{\max }\right)} .
$$

It is well known, however, that the degree of structure present in a process is not quantified by randomness measures, and consequently, measures of statistical or structural complexity are necessary for a better understanding of chaotic time series [48]. There is no universally-accepted definition of complexity. Intuitively, complexity should be related to the amount of structure or the number of patterns present in a system. One would like to have some functional $C[\mathcal{P}]$ adequately capturing the "structuredness" in the same way as Shannon's entropy [44] captures randomness.

The perfect crystal and the isolated ideal gas are two typical examples of systems with minimum and maximum entropy, respectively. However, they are also examples of simple models and, therefore, of systems with zero complexity, as the structure of the perfect crystal is completely described by minimal information (i.e., distances and symmetries that define the elementary cell) and the probability distribution for the accessible states is centered around a prevailing state of perfect symmetry. On the other hand, all of the accessible states of the ideal gas occur with the same probability and can be described by a "simple" uniform distribution. According to López-Ruiz et al. [49] and using an oxymoron, an object, a procedure or system is said to be complex when it does not exhibit patterns regarded as simple. It follows that a suitable complexity measure should vanish both for completely ordered and completely random systems and cannot only rely on the concept of information (which is maximal and minimal for the above-mentioned systems). 
A suitable measure of complexity cannot be made in terms of just "disorder" or "information". It might seem reasonable to propose a measure of "statistical complexity" by adopting some kind of distance to a reference probability distribution, in particular to the uniform distribution $\mathcal{P}_{e}$ [49-51]. This motivates the introduction of the so-called "disequilibrium-distance" $Q\left[\mathcal{P}, \mathcal{P}_{e}\right]$ as a special distance form. In this respect, Rosso and co-workers [51] introduced an effective statistical complexity measure that is able to detect essential details of the dynamics. Then, the wavelet statistical complexity (WSC) measure is defined following the functional product proposed by López-Ruiz et al. [49] (the pdfs involved are given by the probability energy wavelet distribution):

$$
C\left[\mathcal{P}^{(w)}\right]=H\left[\mathcal{P}^{(w)}\right] \cdot Q\left[\mathcal{P}^{(w)}, \mathcal{P}_{e}^{(w)}\right]
$$

where $H \in[0,1]$ is the normalized Shannon entropy given by Equation (10) and the disequilibrium $Q$ is defined in terms of the extensive (in the thermodynamical sense) Jensen-Shannon divergence. Namely,

$$
\begin{aligned}
Q\left[\mathcal{P}^{(w)}, \mathcal{P}_{e}^{(w)}\right] & =Q_{0} \cdot \mathcal{J}\left[\mathcal{P}^{(w)}, \mathcal{P}_{e}^{(w)}\right] \\
& =Q_{0} \cdot\left\{S\left[\frac{\mathcal{P}^{(w)}+\mathcal{P}_{e}^{(w)}}{2}\right]-\frac{1}{2} S\left[\mathcal{P}^{(w)}\right]-\frac{1}{2} S\left[\mathcal{P}_{e}^{(w)}\right]\right\}
\end{aligned}
$$

and $Q_{0}$ is a normalization constant equal to the inverse of the maximum possible value of $\mathcal{J}\left[\mathcal{P}^{(w)}, \mathcal{P}_{e}^{(w)}\right]$, so that $Q \in[0,1]$. $Q_{0}$ is obtained when one of the components of $\mathcal{P}^{(w)}$, say $p_{n}$, is equal to one and the remaining $p_{i}$ are equal to zero.

The Jensen-Shannon divergence quantifies the difference between two (or more) probability distributions and is well-suited to compare the symbol composition between different sequences [52]. The statistical complexity measure value of a system is null and void in the opposite extreme situations of perfect knowledge (perfect crystal) and maximal randomness (ideal gas), whereas a wide range of possible degrees of physical structure does exist between these extreme configurations.

The statistical complexity in Equation (11) is not a trivial function of the entropy, because it measures the interplay between the information stored by the system and the distance from equipartition (the measure of a probabilistic hierarchy between the observed parts) of the probability distribution of its accessible states [49]. Furthermore, a range of possible statistical complexity measure values does exist for a given $H$ value [53], that is $C^{(\min )} \leq C \leq C^{(\max )}$, meaning that additional information related to the dependence structure between the components of the system and the emergence of nontrivial collective behavior is provided by evaluating the statistical complexity. Moreover, it should be noted that statistical complexity fulfills two additional properties required for a suitable definition of complexity in physics [54]: (a) the quantifier must be measurable in different physical systems; and (b) it should enable physical interpretation and comparison between two measurements. Indeed, the definition of complexity in Equation (11) also depends on the scale. For a given system at each scale of observation, a new set of accessible states appears with its corresponding probability distribution, so that complexity changes, and therefore, different values for $H$ and $C$ are obtained.

In order to follow the temporal evolution of the previously-defined information theory quantifiers based on the wavelet transform (relative wavelet energy, normalized wavelet Shannon entropy and wavelet statistical complexity), the analyzed signal is divided into non-overlapping temporal windows, and for each interval, the quantifiers are evaluated. The obtained value is assigned to the central point 
of the time window. In the case of dyadic wavelet decomposition, at resolution level $j$, the number of wavelet coefficients is two-times smaller than in the previous, $j-1$ one. The minimum length of the temporal window will therefore include at least one wavelet coefficient in each scale.

\subsection{Wavelet Leaders and Quantifiers}

In this section, we extend the ODWT methodology by evaluating information quantifiers at wavelet leader-based probability distributions, defined at each signal datum. We have introduced a pointwise wavelet leader Shannon entropy in [32]. In this work we define a pointwise wavelet leader statistical complexity, thereby obtaining pointwise information quantifiers that are capable of capturing irregular structures from signals.

Singularities and irregular structures often carry essential information in a signal or image. For example, in image processing, the contour of objects relates to the discontinuities and singularities in the image. In order to characterize these localized singular structures, it is necessary to quantify the local regularity of the signal. An alternative is to study the pointwise Hölder exponent, which quantifies how rugous or spiky the graph of the function is. A low pointwise Hölder exponent value indicates an irregular point, while the smooth portions of a function have higher exponents.

The pointwise Hölder exponent is defined at each $x_{0} \in \mathbb{R}$, the real number set, in the domain of a locally-bound function $f$, as:

$$
h_{f}\left(x_{0}\right)=\sup _{0 \leq \alpha<+\infty}\left\{\alpha: f \in C^{\alpha}\left(x_{0}\right)\right\}
$$

recalling that a function $f$ is in the class $C^{\alpha}\left(x_{0}\right)$ if there exists $C>0$ and a polynomial $P_{x_{0}}(x)$ of degree less than $\alpha$, such that, near the point $x_{0}$,

$$
\left|f(x)-P_{x_{0}}(x)\right| \leq C\left|x-x_{0}\right|^{\alpha}
$$

In the case $\alpha=0$, we adopt, for convention, that the null polynomial has degree $-\infty$ and $|f(x)| \leq C$.

We illustrate this notion with the following simple examples. The functions $f(x)=|x|^{\alpha}$ and $f(x)=|x|^{\alpha} \sin \left(\frac{\omega}{|x|^{\beta}}\right)$ continuously extended, with $0<\alpha \leq 1, \omega>>1, \beta>0$, have $h_{f}(0)=\alpha$ and $h_{f}(x)=+\infty$, otherwise. This fact shows that the pointwise Hölder exponent captures a singularity in a regular environment. Note that the pointwise Hölder exponent is the usual and most preferable regularity exponent used in signal and image processing [55]. However, other exponents and parameters can be used to extend the local regularity analysis for non-locally bound functions [56,57].

The wavelet transform is an appropriate tool for studying the local regularity. Additional properties are required for the wavelet mother $\psi$. More precisely, we suppose that the admissible mother wavelet $\psi$ is $C^{r}, r \in \mathbb{N}$, with derivatives that have a fast decay, and $\psi$ has $r$ vanishing moments, that is,

$$
\int_{-\infty}^{+\infty} x^{k} \psi(x) d x=0 \quad \text { for } \quad 0 \leq k<r, k \in \mathbb{N} .
$$

If the mother wavelet has $r$ vanishing moments, the wavelet transform can be interpreted as a multiscale differential operator of order $r$. This yields a first relation between the differentiability of $f$ and its wavelet transform decay at fine scales [11]. 
Recalling Equation (3), for $f$, a signal in the space of signals having a finite energy $L^{2}(\mathbb{R})$,

$$
f(x)=\sum_{j \in \mathbb{Z}} \sum_{k \in \mathbb{Z}} c_{j, k} \psi\left(2^{j} x-k\right),
$$

where $c_{j, k}=2^{j / 2}\left\langle f, \psi_{j, k}\right\rangle=2^{j / 2} C_{j, k}$.

There is a direct correlation between the coefficients $c_{j, k}$ and the pointwise Hölder regularity. If $f$ is in the class $C^{\alpha}\left(x_{0}\right)$, it is proven in [58] that the wavelet coefficients of $f$ satisfy, for all $j \geq 0$,

$$
\left|c_{j, k}\right| \leq C 2^{-j \alpha}\left(1+\left|2^{j} x_{0}-k\right|\right)^{\alpha},
$$

for some constant $C$.

S. Jaffard [34] reformulates this property in its simplest formula, characterizing the local regularity in terms of the local suprema of wavelet coefficients: the wavelet leaders. If the mother wavelet $\psi$ is essentially localized on the interval $[0,1]$, then $\psi_{j, k}$ is localized on the dyadic interval $I_{j, k}=\left[\frac{k}{2^{j}}, \frac{k+1}{2^{j}}\right)$, which means that the wavelet coefficient of $f$ in $I_{j, k}$ has information related to this interval.

Then, wavelet leaders of a bounded function $f$ are defined as,

$$
d_{j, k}=\sup _{I_{l, h} \subset 3 I_{j, k}}\left|c_{l, h}\right|
$$

where $3 I_{j, k}=\left[\frac{k-1}{2^{j}}, \frac{k+2}{2^{j}}\right)$ is the dilated interval.

If $x_{0}$ is in the domain of $f, I_{j}\left(x_{0}\right)$ denotes the unique dyadic interval $I_{j, k}$ containing $x_{0}$ for the level $j$. Then, the wavelet leader for $x_{0}$ in the level $j$ is defined as:

$$
d_{j}\left(x_{0}\right)=\sup _{I_{l, h} \subset 3 I_{j}\left(x_{0}\right)}\left|c_{l, h}\right|
$$

Figure 1 illustrates this definition.

Figure 1. A schematic illustration of the definition of wavelet leaders.

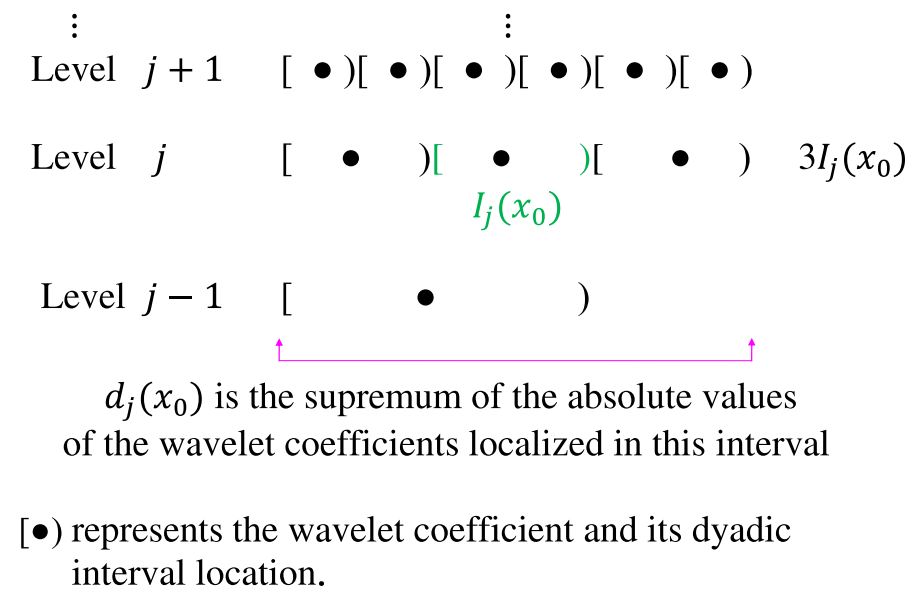

These multiresolution coefficients have been presented in [33], and their hierarchical order yields translation invariant wavelet leader information at each scale $j$, analogous to the CWT. Therefore, leader coefficients are capable of revealing patterns in each scale or successive scales, regardless of 
their temporal position. Furthermore, the wavelet leader information is located in decreasing radius cones as it moves in the scales $j$, reformulating the wavelet characterization of the local regularity and establishing the following properties (proven in [34]).

Let $f$ be a bounded function in $C^{\alpha}\left(x_{0}\right)$, with $\alpha>0$. Then, for all $j>0$,

$$
d_{j}\left(x_{0}\right) \leq C 2^{-j \alpha}
$$

for some constant $C$. Furthermore, if $f$ is uniformly Hölder, i.e., there exists $C>0$ and $0<\epsilon<1$, such that, $\forall x, y \in \mathbb{R}$,

$$
|f(x)-f(y)| \leq C|x-y|^{\epsilon},
$$

then the pointwise Hölder exponent of $f$, defined by Equation (13), can be computed using:

$$
h_{f}\left(x_{0}\right)=\liminf _{j \rightarrow+\infty} \frac{\log \left(d_{j}\left(x_{0}\right)\right)}{\log \left(2^{-j}\right)} .
$$

This property is independent of the wavelet mother election, as long as $\psi$ has the required conditions, $\psi$ has $r$ vanishing moments and $\psi \in C^{r}$ has fast decay derivatives $\psi^{(n)}, 0 \leq n \leq r$, with $r>\alpha$.

By combining Equation (19) and the definition of information theory quantifiers from the previous section, we have defined [32] a pointwise wavelet leader Shannon entropy (WLSS) by using a discrete probability distribution $\mathcal{P}_{x_{0}}^{(w l)}$ at each $x_{0}$ in the domain of a bounded function $f$. That is a pdf based on the wavelet leaders. Recalling that $d_{j}\left(x_{0}\right)$ is the wavelet leader coefficient for $x_{0}$ in the level $j$ defined by Equation (19), this pdf is defined, at each $x_{0}$ in the domain of $f$, as:

$$
\mathcal{P}_{x_{0}}^{(w l)}=\left\{\varrho_{1}, \ldots, \varrho_{j_{\max }}\right\}
$$

such that:

$$
\varrho_{j}=\frac{d_{j}^{2}\left(x_{0}\right)}{\sum_{i=1}^{j_{\max }} d_{i}^{2}\left(x_{0}\right)}
$$

if $d_{j}\left(x_{0}\right) \neq 0$ and $\varrho_{i}=0$, otherwise.

Using Equation (9), the pointwise wavelet leader Shannon entropy at $x_{0} \in \operatorname{Dom}(f)$ is:

$$
S_{f}\left(x_{0}\right)=S\left[\mathcal{P}_{x_{0}}^{(w l)}\right]=-\sum_{j=1}^{j_{\max }} \varrho_{j} \log _{2}\left(\varrho_{j}\right)
$$

If $\varrho_{j}=0$, it is defined $\varrho_{j} \log _{2}\left(\varrho_{j}\right)=0$. In analogous form, by use of Equation (11), we can also introduce the wavelet leader statistical complexity (WLSC) at each $x_{0}$ in the domain of a bounded function $f$ as,

$$
C_{f}\left(x_{0}\right)=C\left[\mathcal{P}_{x_{0}}^{(w l)}\right]=H\left[\mathcal{P}_{x_{0}}^{(w l)}\right] \cdot Q\left[\mathcal{P}_{x_{0}}^{(w l)}, \mathcal{P}_{x_{0}, e}^{(w l)}\right]
$$

From Equation (22), the following inverse relation between the wavelet leader entropy and the pointwise Hölder exponent has been proven in [32]:

Let $h=h_{f}\left(x_{0}\right)$. If $f \in \mathcal{C}^{h}\left(x_{0}\right)$ and $f$ is uniformly Hölder, then, for each $a>1$, there exists a resolution level $m$, such that:

$$
4^{-(m a-1) h} \log _{2}\left(m 4^{-(m a-1) h}\right) \leq S_{f}\left(x_{0}\right) \leq \log _{2}(m)
$$


Then, it is established that $S_{f}\left(x_{0}\right)$ takes values close to its maximum $\log _{2}(m)$ when $h_{f}\left(x_{0}\right)$ takes values close to zero. Otherwise, recalling that $Q\left[\mathcal{P}_{x_{0}, e}^{(w l)}, \mathcal{P}_{x_{0}, e}^{(w l)}\right]=0$ and $S\left[\mathcal{P}_{x_{0}, e}^{(w l)}\right]=\log _{2}(m)$, then $C_{f}\left(x_{0}\right)$ takes values close to zero when $S_{f}\left(x_{0}\right)$ takes values close to its maximum $\log _{2}(m)$. Consequently, the irregular structures of the signal are related on the highest values of $S_{f}\left(x_{0}\right)$ and the lowest values of $C_{f}\left(x_{0}\right)$.

We propose the following steps for estimating these pointwise quantifiers from data:

1. Via the Mallat algorithm [40], compute $c_{j, k}$ for the resolution levels $j=1, \cdots, j_{\max }$ (see Equation (16)). Considering the data series $\{f(n): n=1, \cdots, N\}$ at the finest level, then $j_{\max } \leq \log _{2}(N)$.

2. From the definition given by Equation (19), estimate the wavelet leaders $\left(d_{j}(n)\right)_{j=1, \cdots, j_{\max }}$ using the following equation:

$$
d_{j}(n)=\sup \left\{\left|c_{l, h}\right|: I_{l, h} \subset 3 I_{j}(n), 1 \leq l \leq j_{\max }\right\}
$$

3. Calculate $S_{f}(n)$ and $C_{f}(n)$ for the resolution level $j_{\text {max }}$, using Equations (24)-(26), entering the Jensen-Shannon divergence (Equation (12)) in the $Q$ definition.

\section{3. qEEG Analysis of a Tonic-Clonic Epileptic Signal}

\subsection{Clinical Data and the Experimental Setup}

A scalp EEG signal is essentially a nonstationary time series that presents artifacts due to the electrooculogram (EOG), electromyogram (EMG) and electrocardiogram (ECG), among others [1,59-61]. Sometimes, artifacts present during a few seconds and can be obviated, because they obscure only a small portion of the EEG. In other cases, almost the total signal appears obscured by them, and very little information about the underlying brain activity can be extracted.

Epileptic seizures are symptoms of brain dysfunction produced by a synchronous or paroxysmal discharge of a group of neurons located in the cerebral cortex. Those classified as "secondary generalized epileptic seizures" usually begin with abnormal neuronal discharges, focused, capable of spreading to the rest of the brain cells and lead to a tonic-clonic epileptic seizure. They last about 1 to 2 min and begin with the loss of individual conscience.

A tonic-clonic epileptic seizure (TCES) is characterized by violent muscle contractions. Initial massive tonic spasms are replaced seconds later by the clonic phase with violent flexor movements and characteristic rhythmic spasms towards the end of the seizure [1]. In these seizures, artifacts related to muscle contractions, are especially troublesome, because they reach very high amplitudes. In fact, not only do they limit the traditional visual analysis to the pre- and post-seizure periods, they also restrict the application of some mathematical methods.

Analysis of the brain activity during this kind of seizure has been previously performed only in special circumstances, such as in patients treated with curare (an inhibitor of muscle responses) [62,63] or by eliminating the high-frequency muscle activity with the use of traditional filters [64]. Gastaut and Broughton $[62,63]$ described a frequency pattern during a tonic-clonic epileptic seizure from patients with muscle relaxation from curarization and artificial respiration. 
After a short period (which may be as short as 1-3 s) characterized by phase desynchronization, they found an epileptic recruiting rhythm (ERR) at about $10 \mathrm{~Hz}$ with a rapidly increasing amplitude dominating the EEG. Later, as the seizure ends, there is a progressive increase of the lower frequencies associated with the clonic phase. About $10 \mathrm{~s}$ after the seizure onset, lower delta frequencies $(0.5-3.5 \mathrm{~Hz})$ are observed to gradually diminish in their activity. The clonic activity corresponds with generalized polyspike bursts at each myoclonic jerk. Very slow irregular delta activity dominates the EEG then, accompanied with a gradual frequency increase of the theta $(3.5-7.5 \mathrm{~Hz})$ and alpha bands $(7.5-12.5 \mathrm{~Hz})$, indicative of the end of the seizure.

In Figure 2, we present a scalp EEG record, corresponding to a tonic-clonic epileptic seizure recorded in the right central location (C4 channel).

Figure 2. Scalp EEG signal for a tonic-clonic epileptic seizure (TCES), recorded at the central right location, the $\mathrm{C} 4$ channel. The vertical lines mark the following transitions: The seizure starts at $\mathbf{T I}=80 \mathrm{~s}$ and the clonic phase at $\mathbf{T} 2=125 \mathrm{~s}$. The seizure ends at $\mathbf{T F}=$ $155 \mathrm{~s}$. Notice that the dramatic transition from rigidity (tonic stage) to convulsions (clonic stage) around $\mathrm{T} 2=125 \mathrm{~s}$ is not clearly discernible. $\mathbf{T} 1=90 \mathrm{~s}$ and $\mathbf{T} 3=145 \mathrm{~s}$ mark the expected beginning and end of the epileptic recruiting rhythm (ERR).

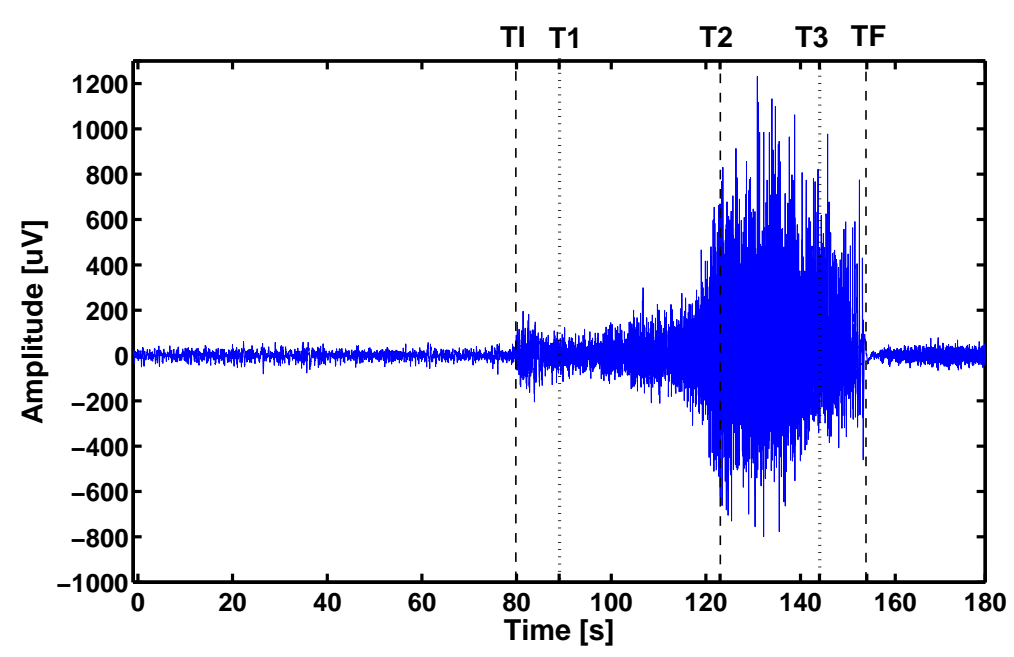

We chose this electrode, in agreement with the team of physicians, after visual inspection of the pertinent EEG records, as it was the one with the minimum amount of artifacts. This record was obtained from a 39-year-old female patient with a diagnosis of pharmacoresistant epilepsy (temporal lobe epilepsy). The localized source of seizure is left temporal, and the patient presents no other accompanying disorders [13,14]. Informed consent from the patient was obtained beforehand. Antiepileptic drugs were gradually reduced in order to precipitate seizures. The time intervals of the pre-seizure stage, which present artifacts (ocular and other movements, etc.) were marked by the team of physicians and were excluded in the subsequent analysis.

Scalp and sphenoidal electrodes with a bimastoideal reference were applied following the 10/20 international system, and a seizure button manually activated by the patient was also available. The signal was digitized at $409.6 \mathrm{~Hz}$ through a 12 bit A/D converter and filtered with an anti-aliasing eight-pole low-pass Bessel filter, with a cutoff frequency of $50 \mathrm{~Hz}$. Afterwards, the signal was digitally filtered with a $1-50 \mathrm{~Hz}$ bandwidth Butterworth filter and stored, after decimation, at $\omega_{s}=102.4 \mathrm{~Hz}$, in a PC 
hard drive. Recordings were performed under video control in order to have an accurate determination of the different stages of the seizure. The different stages of EEG signals were determined by the team of physicians. Off-line analysis was performed with characterization of semiological features. When possible, the onset and definition of the anatomical focus for each event were timed. The analysis for each event included $60 \mathrm{~s}$ of EEG before the seizure onset and $120 \mathrm{~s}$ of seizure and post-seizure phases.

In this record (Figure 2), the pre-seizure is characterized by a signal of $50 \mu \mathrm{V}$. The epileptic seizure starts at $\mathbf{T I}=80 \mathrm{~s}$, with a "discharge" of slow waves superposed by fast ones with a lower amplitude. This discharge lasts $\sim 8 \mathrm{~s}$ and has a mean amplitude of $100 \mu \mathrm{V}$. Afterward, the seizure spreads, making the analysis of the EEG more complicated due to muscle artifacts. However, it is possible to establish the beginning of the clonic phase at around $\mathrm{T} 2=125 \mathrm{~s}$ and the end of the seizure at $\mathrm{TF}=155 \mathrm{~s}$, where there is an abrupt decay of the signal amplitude. The start and ending epileptic recruiting rhythm are expected at $\mathbf{T} 1 \sim 75 \mathrm{~s}$ and $\mathbf{T} 3 \sim 110 \mathrm{~s}$, according to Gastaut and Broughton [62,63].

EEG spectral analysis is traditionally performed by studying different frequency bands with well-defined boundaries. Some small variations can be found, according to the particular experiment under consideration. The absolute and relative intensities of these bands are usually analyzed and correlated with different pathologies. In this work, we define fourteen frequency bands for an appropriate ODWT analysis within the multiresolution scheme to be used. We have denoted these band resolution levels as $\mathrm{B}_{j}(j=1, \cdots, 14)$. Their frequency limits, wavelet levels, as well as their correspondence with traditional EEG frequency bands are given in Table 1. As mentioned above, in the present work, we use an orthogonal cubic B-spline function as a mother wavelet. We eliminate the high frequency muscle activity by using wavelet analysis. We define $j_{\max }=\log _{2} N \cong 14$ ( $N=18,432$ being the total number of data in our time series) frequency bands for an appropriate wavelet analysis within the multiresolution scheme to be used. Their frequency limits are given by $2^{j-j_{\max }-2} \omega_{s} \leq|\omega| \leq 2^{j-j_{\max }-1} \omega_{s}$, where $\omega_{s}=102.4 \mathrm{~Hz}$ is the sample frequency. Their correspondence with traditional EEG frequency bands is given in Table 1.

Table 1. Frequency boundaries (in $H z$ ) associated with the different orthogonal discrete wavelet transform (ODWT) resolution wavelet levels $j$ with sample frequency $\omega_{s}=102.4 \mathrm{~Hz}$ and time series length $N=18,432$. The traditional EEG frequency bands correspond to the following frequencies: $\delta(0.5-3.5 \mathrm{~Hz}) ; \theta(3.5-7.5 \mathrm{~Hz}) ; \alpha(7.5-12.5 \mathrm{~Hz}) ; \beta(12.5-30.0 \mathrm{~Hz})$; $\gamma(>30.0 \mathrm{~Hz})$.

\begin{tabular}{ccccc}
\hline $\mathbf{B}_{\boldsymbol{j}}$ & $\boldsymbol{\omega}_{\boldsymbol{m i n}}(\mathrm{Hz})$ & $\boldsymbol{\omega}_{\max }(\mathrm{Hz})$ & $\boldsymbol{j}$-Level & EEG Band \\
\hline $\mathrm{B}_{14}$ & 25.6 & 51.2 & 14 & $\beta, \gamma$ \\
$\mathrm{B}_{13}$ & 12.8 & 25.6 & 13 & $\beta$ \\
$\mathrm{B}_{12}$ & 6.4 & 12.8 & 12 & $\theta, \alpha$ \\
$\mathrm{B}_{11}$ & 3.2 & 6.4 & 11 & $\theta$ \\
$\mathrm{B}_{10}$ & 1.6 & 3.2 & 10 & $\delta$ \\
$\mathrm{B}_{9}$ & 0.8 & 1.6 & 9 & $\delta$ \\
\hline
\end{tabular}

We limit the evaluation of different wavelet quantifiers defined above (see Sections 2.2 and 2.3) to the middle and low frequencies (frequency bands $\mathrm{B}_{9}$ to $\mathrm{B}_{12}, 0.8-12.8 \mathrm{~Hz}$ ). We did not consider the 
contributions from bands $\mathrm{B}_{13}(25.6-12.8 \mathrm{~Hz})$ and $\mathrm{B}_{14}(51.2-25.6 \mathrm{~Hz})$ with wavelet resolution levels $j=13$ and $j=14$, respectively, both containing high frequency artifacts related to muscle activity that blurred the EEG [64]. Although high frequency brain activity is also eliminated, their contributions during the seizure stage are not as important as those of the middle and low frequencies, as has been previously shown in [18]. In fact, the inclusion of these high frequency bands, which present a very disordered activity, makes the mathematical distinction between pre-seizure and during the seizure EEG (mean values and standard deviation over time intervals) more difficult. In particular, it is not possible to define and quantify the epileptic recruitment rhythm correctly.

In Figure 3, we display the EEG signal without the contribution of the frequency bands $B_{13}$ and $B_{14}$ and the EEG signal reconstructed using wavelet resolution levels $j=9$ to $j=12$. In order to study the temporal evolution of the information theory quantifiers based on ODWT coefficients, the analyzed signal is divided into non-overlapping temporal windows of length $L=2.5 \mathrm{~s}$ each $(N=256$ data), and for each interval, the quantifiers are evaluated.

Figure 3. Noise-free signal, reconstructed from wavelet frequency bands $B_{9}$ to $B_{12}$ of the scalp EEG signal for a TCES, recorded at the central right location, the $\mathrm{C} 4$ channel. The vertical lines indicate the following transitions: The seizure starts at $\mathbf{T I}=80 \mathrm{~s}$ and the clonic phase at $\mathbf{T} 2=125 \mathrm{~s}$. The seizure ends at $\mathbf{T F}=155 \mathrm{~s}$. $\mathbf{T} 1=90 \mathrm{~s}$ and $\mathrm{T} 3=145 \mathrm{~s}$ mark the expected beginning and end of the epileptic recruiting rhythm (ERR).

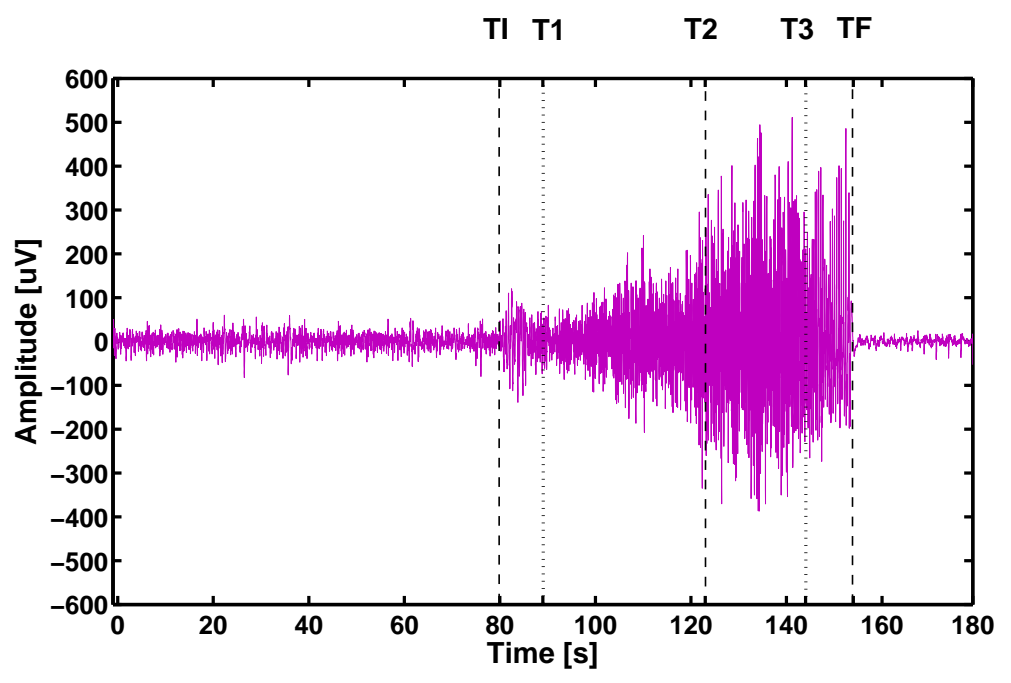

\subsection{Analysis Based on a Set of ODWT Coefficients}

Figure 4 displays the relative wavelet energies (RWE) without electromyographic contributions $\left(\mathrm{B}_{9}\right.$ to $\mathrm{B}_{12}$ ) for the EEG tonic-clonic epileptic record shown in Figure 2.

We see that the pre-seizure phase is characterized by a signal dominance of low rhythms (pre-seizure: $\left.\left[\rho_{9}+\rho_{10}\right] \sim 50 \%\right)$. The seizure starts at $\mathbf{T I}=80 \mathrm{~s}$ with a discharge of slow waves superimposed with low voltage fast activity. This discharge lasts approximately $8 \mathrm{~s}$ and produces a marked rise in low frequency bands (delta activity), which reaches $\left[\rho_{9}+\rho_{10}\right] \sim 80 \%$ of the RWE. From $90 \mathrm{~s}$ onwards, the low frequency activity, represented by $\mathrm{B}_{9}$ and $\mathrm{B}_{10}$, decreases abruptly to values lower than $10 \%$, 
and the other frequency bands (alpha and theta activity, represented by $\mathrm{B}_{11}$ and $\mathrm{B}_{12}$ frequency bands, respectively) alternatively dominate.

Figure 4 also shows that the start of the clonic phase is correlated with a rise of $B_{11}$, and after $140 \mathrm{~s}$, when clonic discharges begin to separate further, $\mathrm{B}_{10}$ rises up again until the end of the seizure, when $\mathrm{B}_{9}$ also increases very quickly and both frequencies bands are dominant. The RWE contribution of frequency bands $\mathrm{B}_{9}$ and $\mathrm{B}_{10}\left(\rho_{9}\right.$ and $\left.\rho_{10}\right)$ maintains this behavior throughout the post-seizure phase. We could conclude from this example that the seizure was dominated by the middle frequency bands $\mathrm{B}_{11}$ and $\mathrm{B}_{12}$ (alpha and theta rhythms, 3.2-12.8 Hz), with a corresponding abrupt activity decrease in the low frequency bands $\mathrm{B}_{9}$ and $\mathrm{B}_{10}$ (delta rhythm, 0.8-3.2 Hz). Clearly, this behavior can be associated with the putative "epileptic recruiting rhythm" (ERR) described by Gastaut and Broughton [63] and represented by the time interval between vertical lines T1 and T3 in Figure 4.

Figure 4. Time evolution of the relative wavelet energy (RWE) corresponding to the EEG noise-free signal (Figure 3), for the frequency bands $\mathrm{B}_{9}, \mathrm{~B}_{10}, \mathrm{~B}_{11}$ and $\mathrm{B}_{12}$. The vertical lines indicate the following transitions: The seizure starts at $\mathrm{TI}=80 \mathrm{~s}$ and the clonic phase at $\mathrm{T} 2=125 \mathrm{~s}$. The seizure ends at $\mathbf{T F}=155 \mathrm{~s}$. $\mathbf{T} 1=90 \mathrm{~s}$ and $\mathrm{T} 3=145 \mathrm{~s}$ mark the expected beginning and end of the epileptic recruiting rhythm (ERR).

TI T1

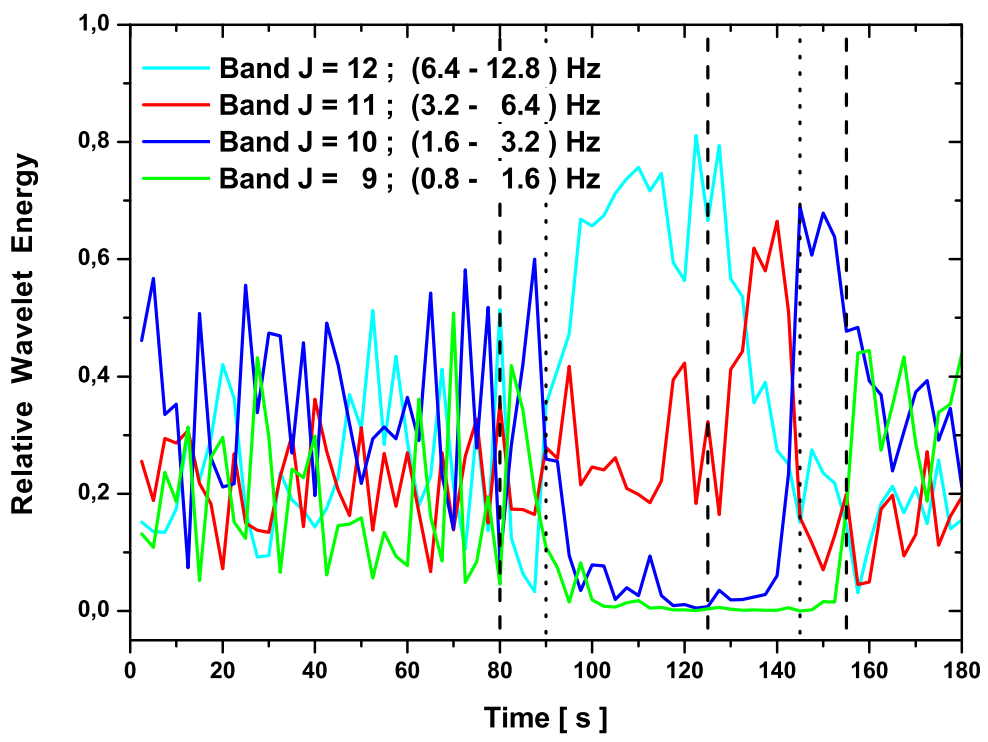

The normalized Shannon wavelet entropy (normalized SWS) as a function of time is presented in Figure 5. In Figure 5, one line represents the time evolution of the normalized SWS (frequency bands $\mathrm{B}_{9}$ to $\mathrm{B}_{14}$ are included), while the other line corresponds to results that ignore the contributions coming from high frequency bands $\left(\mathrm{B}_{13}\right.$ and $\left.\mathrm{B}_{14}\right)$, which mainly contain electromyographic activity.

It is interesting to observe the behavior of the normalized SWS during the first $10 \mathrm{~s}$ following the seizure onset. We see that in this time interval, the normalized SWS exhibits increasing values if the contribution of wavelet frequency bands $\mathrm{B}_{9}$ to $\mathrm{B}_{14}$ are included. A comparison is to be made with normalized SWS values in the pre-seizure stage. If the wavelet frequency bands $B_{13}$ and $B_{14}$ (bands that mainly reflect muscular activity) are not included, the mean normalized SWS value is lower than that for the pre-seizure one. Thus, the behavior of the normalized SWS after the onset of the 
seizure is compatible with an increase in the degree of disorder of the system, induced by a high frequency activity. Superimposed low and medium frequency activities, however, are responsible for the "remaining-signal's more orderly behavior". The decreasing values of the normalized SWS after $\mathbf{T} 1=90 \mathrm{~s}$ (in both cases, with and without inclusion of high frequency bands) are indicative that the system presents a tendency to a more ordered behavior. This tendency is better appreciated without muscle activity. The normalized SWS behavior is clearly correlated with the ERR described by Gastaut and Broughton [63] and represented by the time interval between vertical lines T1 and T3 in the figure. Moreover, note that the normalized SWS in the last case adopts a minimum value around T2 $=125 \mathrm{~s}$, coincident with the beginning of the clonic phase. The peak observed in the normalized SWS at T3 $\cong 145 \mathrm{~s}$ could be associated with the disappearance of the ERR, again in agreement with Gastaut and Broughton's description. After this point, the normalized SWS displays increasing values until TF $=155 \mathrm{~s}$, which is defined as the seizure's ending time. Quantitatively, the normalized SWS for the post-seizure stage presents almost constant values, comparable to those obtained for the pre-seizure stage.

Figure 5. Temporal evolution of the normalized Shannon wavelet entropy (normalized SWS). One line represents the normalized SWS when frequency bands $\mathrm{B}_{9}$ to $\mathrm{B}_{14}$ are included, while the other corresponds to results that ignore the contributions coming from high frequency bands, $\mathrm{B}_{13}$ and $\mathrm{B}_{14}$, which mainly contain electromyographic activity. The vertical lines indicate the following transitions: The seizure starts at $\mathbf{T I}=80 \mathrm{~s}$ and the clonic phase at $\mathbf{T} 2=125 \mathrm{~s}$. The seizure ends at $\mathbf{T F}=155 \mathrm{~s}$. $\mathbf{T} 1=90 \mathrm{~s}$ and $\mathbf{T} 3=145 \mathrm{~s}$ mark the expected beginning and end of the epileptic recruiting rhythm (ERR).

TI T1 T2 T3 TF

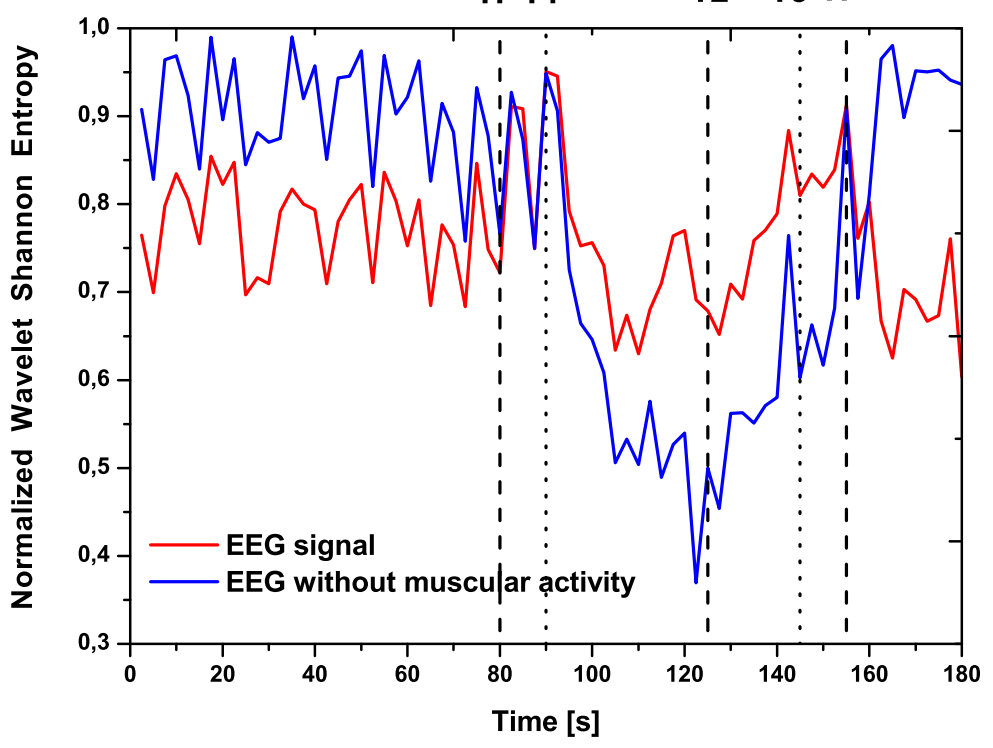

Note that the absolute minimum value of the normalized entropy is to be found in the vicinity of $\sim 125 \mathrm{~s}$, in agreement with the medical diagnosis: in that neighborhood, one encounters the tonic-clonic "phase transition". For normalized SWS, two relative maxima are observed at $\sim 145 \mathrm{~s}$ and $\sim 155 \mathrm{~s}$. As stated above, these times are associated with (i) the end of the epileptic recruiting rhythm and (ii) the end of the epileptic seizure, respectively. Changes in the EEG series around $125 \mathrm{~s}$ (the transition 
from the tonic to clonic stage) are the result of a mechanism entirely different from the one that produces variations at $145 \mathrm{~s}$ and $155 \mathrm{~s}$ (neuronal "fatigue", i.e., both a diminishing of the neuronal firing rate and a preponderance of inhibitory mechanisms become critical factors underlying the seizure's end).

With reference to the normalized SWS as a measure of the degree of order/disorder, we are in a position to assert that the normalized SWS presents lower values in the seizure than in the pre-seizure phase. Summing up, one can associate a more robust degree of order to the EEG activity during the seizure phase than during the pre- and post-seizure stages, compatible with a dynamic process of synchronization in the brain activity. This behavior may be thought as induced by a hypothetical epileptic focus, which generates the ERR observed.

In Figure 6, the time evolution corresponding to wavelet statistical complexity (WSC) is depicted for the EEG record of Figure 2. These lines represent the values obtained when frequency bands $\mathrm{B}_{13}$ and $\mathrm{B}_{14}$ are included or left out in their evaluation.

Figure 6. Temporal evolution of the wavelet statistical complexity (WSC). One line represents the time evolution of the normalized WSC when frequency bands $\mathrm{B}_{9}$ to $\mathrm{B}_{14}$ are included, while the other corresponds to results that ignore contributions coming from high frequency bands, $\mathrm{B}_{13}$ and $\mathrm{B}_{14}$, which mainly contain electromyographic activity. The vertical lines indicate the following transitions: The seizure starts at $\mathrm{TI}=80 \mathrm{~s}$ and the clonic phase at $\mathbf{T} 2=125 \mathrm{~s}$. The seizure ends at $\mathbf{T F}=155 \mathrm{~s} . \mathbf{T} 1=90 \mathrm{~s}$ and $\mathbf{T} 3=145 \mathrm{~s}$ mark the expected beginning and end of the epileptic recruiting rhythm (ERR).

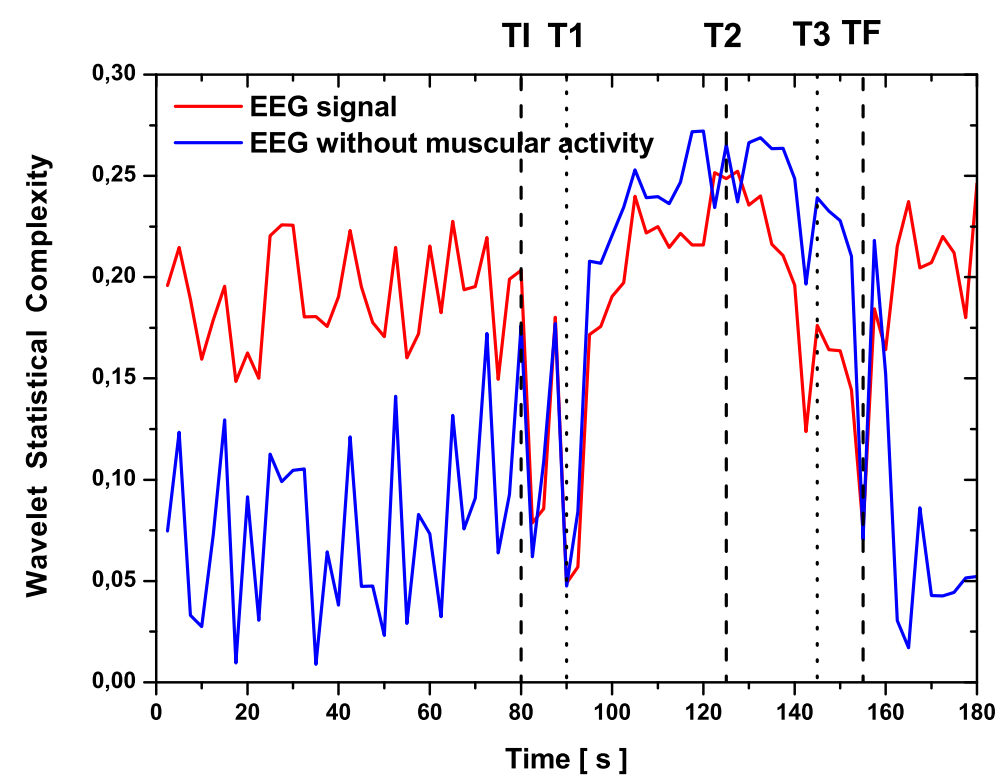

The WSC exhibits almost constant mean values (they resemble each other) in pre-seizure and seizure stages when the analysis includes the frequency bands $\mathrm{B}_{13}$ and $\mathrm{B}_{14}$. Excluding these two bands yields quite different behavior, especially in the time interval identified with the ERR. There, the complexity values are significantly bigger than in the pre- and post-seizure period.

What is significant here is that the time interval from $90 \mathrm{~s}$ to $145 \mathrm{~s}$, associated with the ERR, reveals a great degree of order (low entropy) together with maximum statistical complexity. Order and complexity are seen to coexist. In addition, the wavelet statistical complexity $C$ falls in the vicinity of 
$125 \mathrm{~s}$, corresponding to the tonic-clonic transition. This $C$ "dip" is associated with a marked normalized SWS diminution, opposite of what happens around either $140 \mathrm{~s}$ (disappearance of the ERR) or $155 \mathrm{~s}$ (seizure end time), where the normalized SWS values grow. It seems that a different dynamical regime is obtained at $125 \mathrm{~s}$ vis-á-vis the one at $140 \mathrm{~s}$ or $155 \mathrm{~s}$.

We conclude that muscular activity destroys the underlying neuronal complexity. This is to be expected, of course, but it constitutes an indication regarding how reliable our wavelet statistical complexity measure really is.

[14] presents a more quantitative analysis of the observed behavior of the relative wavelet energy and its relation with the description of ERR, as well as the behavior of normalized Shannon wavelet entropy (SWS) and wavelet statistical complexity (SWC) during tonic-clonic epileptic seizure. In particular, in this reference, 20 tonic-clonic epileptic seizures corresponding to eight patients were analyzed, confirming the behavior of the information theory quantifiers described previously.

\subsection{Analysis Based on a Set of Wavelet Leader Coefficients}

The EEG signal displayed in Figure 2 has been obtained from the original EEG signal record at the C4 electrode, having 18,432 data. The wavelet decomposition can be carried out over all resolution levels $j=1$ to $j_{\max }=14 \leq \log _{2}(18,432)$. For the first approach, we do not consider the contributions from the levels $j=13$ and $j=14$ associated with high frequency muscle activity at computing the probability distribution $\mathcal{P}_{x_{0}}$ in Formula (23).

Figure 7. Wavelet leader Shannon entropy temporal evolution of the filtered signal, without the inclusion of frequency bands containing muscle activity. The vertical lines indicate the following transitions: The seizure starts at $\mathbf{T I}=80 \mathrm{~s}$ and the clonic phase at $\mathbf{T} 2=125 \mathrm{~s}$. The seizure ends at $\mathbf{T F}=155 \mathrm{~s}$. $\mathbf{T} 1=90 \mathrm{~s}$ and $\mathbf{T} 3=145 \mathrm{~s}$ mark the expected beginning and end of the epileptic recruiting rhythm (ERR).

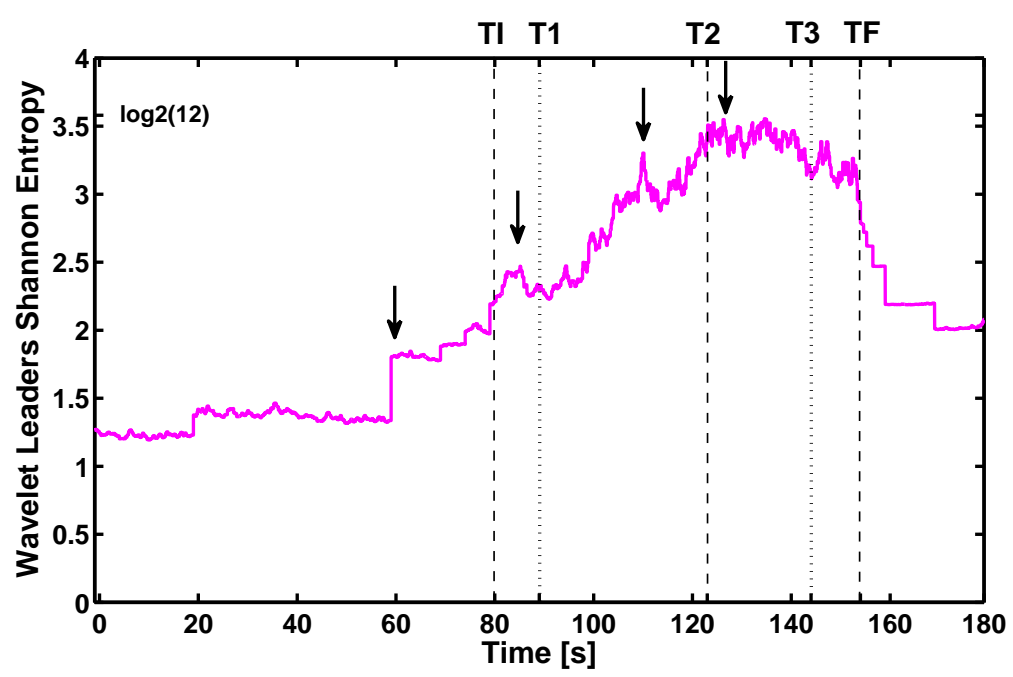

Although the traditional EEG analysis and information quantifiers based on ODWT coefficients are related to the resolution levels $j=9$ to $j=12$ given in Table 1, we examine the resolution levels $j=1$ to 12 to estimate the wavelet leaders in Equation (28), since considering few resolution levels does not 
reveal the local regularity of the signal. The time evolution for the pointwise wavelet Leader Shannon entropy (WLSS) and the pointwise wavelet leader statistical complexity (WLSC) corresponding to the filtered EEG signal, displayed in Figure 3, are shown in Figures 7 and 8.

Furthermore, the time evolution of the pointwise Hölder exponent is displayed in Figure 9, computed from Equation (22). We have supposed that:

$$
\log \left(d_{j}(n)\right) \approx \log (C)+h_{f}(n) \log \left(2^{-j}\right) \quad j=1, \cdots, 12,
$$

and used a linear regression to estimate the pointwise Hölder exponent $h_{f}(n)$. There are several techniques to estimate the pointwise Hölder exponent, and this one is an efficient alternative; see [65] and [55] for further information on this topic.

Figure 8. Wavelet leader statistical complexity temporal evolution of the filtered signal, without the inclusion of frequency bands containing muscle activity. The vertical lines indicate the following transitions: The seizure starts at $\mathbf{T I}=80 \mathrm{~s}$ and the clonic phase at $\mathbf{T} 2=125 \mathrm{~s}$. The seizure ends at $\mathbf{T F}=155 \mathrm{~s}$. $\mathbf{T} 1=90 \mathrm{~s}$ and $\mathbf{T} 3=145 \mathrm{~s}$ mark the expected beginning and end of the epileptic recruiting rhythm (ERR).

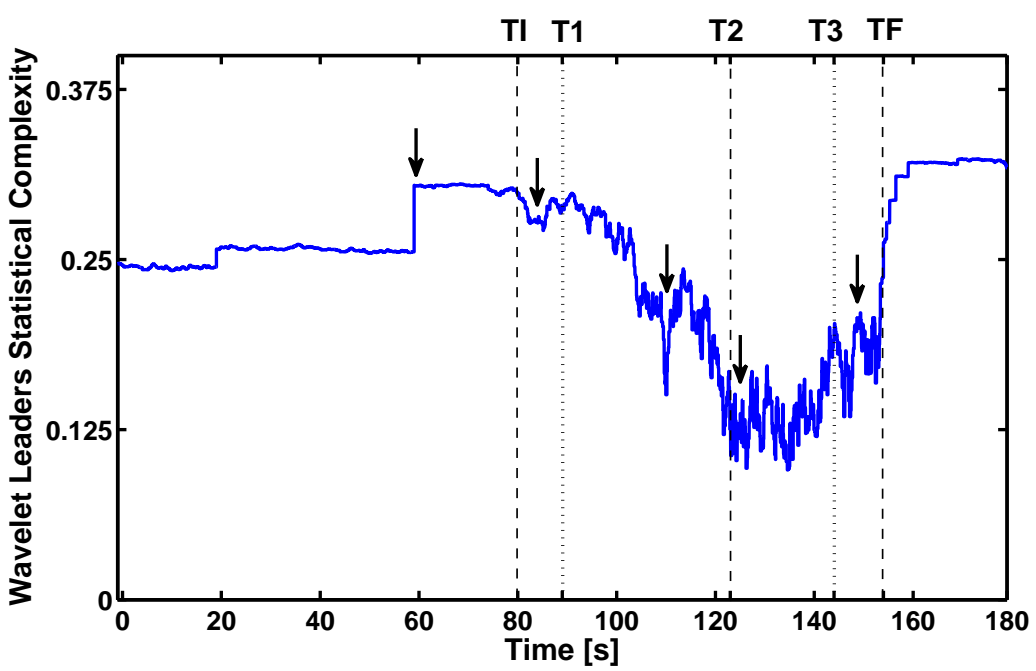

As shown in Equation (27), the WLSS function reaches its relative maximum values when the pointwise Hölder exponents approach zero, which indicate the highly irregular points. The WLSS reaches its absolute maximum when all values of the wavelet leader throughout all of the levels $j$ are equal, for a point $x_{0}$. However, in the case of a natural series, it is practically impossible, so that the maximum corresponds to the position of highest irregularity. In the same sense, this choice in the construction of the pdf also determines that the WLSC reaches minimum values at the points of greatest irregularity. Then, the range variation of the wavelet leader-based quantifiers, the wavelet leader entropy (WLSS) and wavelet leader complexity (WLSC) are the inverse of those quantifiers based on ODWT, that is the wavelet Shannon entropy (SWS) and wavelet statistical complexity (WSC), respectively.

Values of the WLSS near the maximum $\log _{2}(12)$ indicate an irregularity in the signal; otherwise, the lowest values of the WLSC also indicate this fact. It is possible to observe an increase of the WLSS during the TCES, and conversely, the values of the pointwise Hölder exponent and the WLSC fall during the TCES. The maximum values of the WLSS are between T2 and T3, while the pointwise Hölder 
exponent and WLSC take the minimum values. The inverse relation between the WLSS and the Hölder exponents can also be observed, coinciding with the result proven in [32].

Comparing Figures 7-9, it can be noted that WLSS and WLSC distinguish the event analyzed more precisely than the pointwise Hölder exponent, which also takes values concentrated in the interval $[0.20,0.50]$ at the first $60 \mathrm{~s}$, before the beginning of the epileptic seizure, as well as taking these values during the epileptic seizure.

Figure 9. Pointwise Hölder exponent time evolution of the filtered signal, without the inclusion of frequency bands containing muscle activity. The vertical lines indicate the following transitions: The seizure starts at $\mathbf{T I}=80 \mathrm{~s}$ and the clonic phase at $\mathbf{T} 2=125 \mathrm{~s}$. The seizure ends at $\mathbf{T F}=155 \mathrm{~s} . \mathrm{T} 1=90 \mathrm{~s}$ and $\mathrm{T} 3=145 \mathrm{~s}$ mark the expected beginning and end of the epileptic recruiting rhythm (ERR).

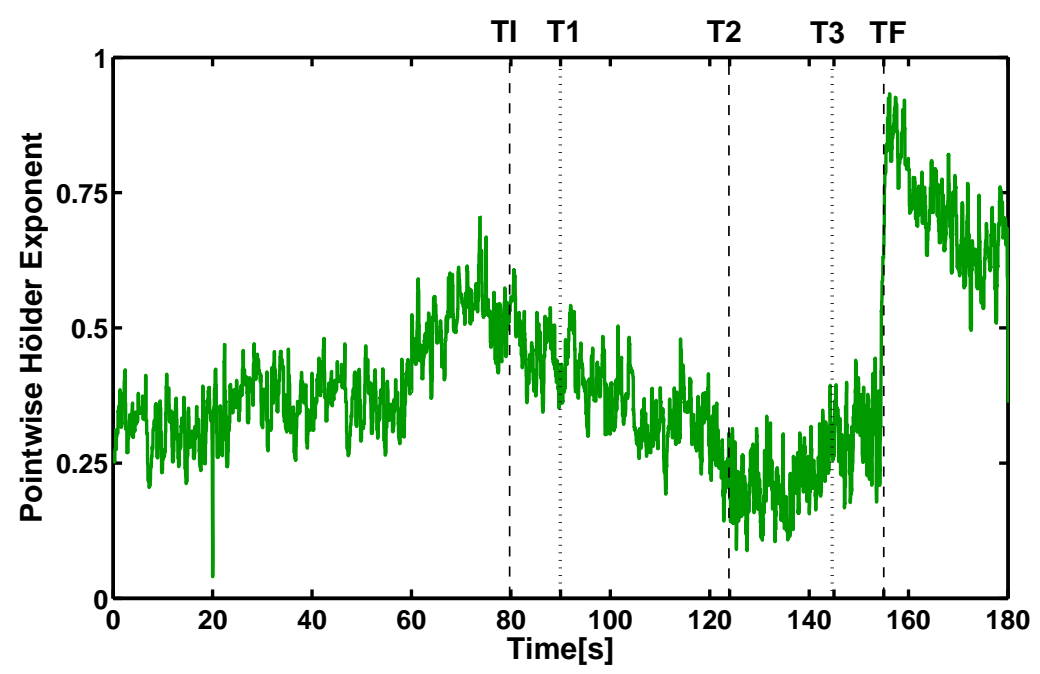

As in Section 3.2, we have marked with dashed lines the times corresponding to the seizure beginning and end, and the dotted lines comprise the time when the ERR is expected. Note that in the pre-seizure (from 0 to $80 \mathrm{~s}$ ), there are two jumps in the values of the wavelet leader entropy quantifier (Figure 7): the first one around $\sim 20 \mathrm{~s}$ and the other around $60 \mathrm{~s}$. Around $85 \mathrm{~s}$, a local maximum is observed a little earlier than the minimum of SWS, which happens at $\sim 90 \mathrm{~s}$. However, the WLSS is growing steadily from $\sim 90 \mathrm{~s}$. The point $\sim 115 \mathrm{~s}$ is a local extremum (maximum in WLSS and minimum in the WLSC) that corresponds to the transition from the tonic to clonic stage. Note that in the figure of WLSS, two relative maxima at $\sim 145 \mathrm{~s}$ and $\sim 155 \mathrm{~s}$ also appear. These events can be associated with (i) the end of the epileptic recruiting rhythm and (ii) the end of the epileptic seizure end, respectively.

It is remarkable to note that wavelet leader-based quantifiers are not affected with a marked change when we calculate the probability distribution $\mathcal{P}_{x_{0}}$ in Equation (23), including the 14 resolution levels for the original signal (Figure 2) and the filtered signal (Figure 3). Figure 10 displays the WLSS evolutions, including the wavelet leaders at 14 resolution levels for the original signal and the filtered signal. On the other hand, Figure 7 shows the WLSS evolution considering 12 levels of resolution for the filtered signal, without considering the wavelet leaders at the resolution levels related to muscle activity. If we compare both figures, we can observe that they show a similar pattern. Nevertheless, the temporal evolution of WLSS for the filtered signal (Figure 10) distinguishes more precisely the period between T2 and T3. 
Figure 10. Temporal evolution of the wavelet leader Shannon entropy for the original signal corresponding to tonic-clonic epileptic EEG record. The vertical lines indicate the following transitions: The seizure starts at $\mathbf{T I}=80 \mathrm{~s}$ and the clonic phase at $\mathbf{T} 2=125 \mathrm{~s}$. The seizure ends at $\mathbf{T F}=155 \mathrm{~s} . \mathrm{T} 1=90 \mathrm{~s}$ and $\mathrm{T} 3=145 \mathrm{~s}$ mark the expected beginning and end of the epileptic recruiting rhythm (ERR).

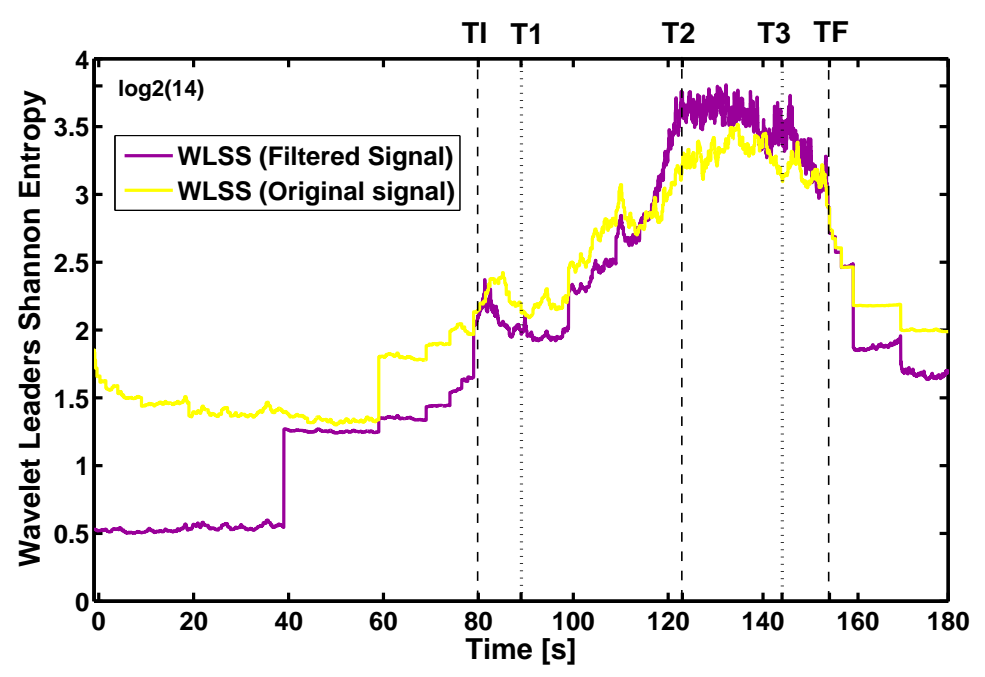

\subsection{Discussion}

We have reviewed the methodology based on ODWT coefficients in the quantitative analysis of EEG epileptic records and, in particular, on tonic-clonic epileptic seizures. The "epileptic recruiting rhythm" (ERR) observed during the development of a seizure is well described in terms of the relative wavelet energy. In addition, we have shown that the use of a set of wavelet leader coefficients enables a more precise time localization in the time description of the dynamical changes in the EEG signal.

One could thus reasonably conjecture that tonic-phase spasms were "answers" to brain oscillations, generated with high frequencies. Due to the fact that muscles cannot contract in such a rapid fashion, muscle activity gets restricted to tonic contraction, until the frequency of brain oscillations becomes slow enough for the muscle to be capable of oscillating in resonance with it [66].

One important point to emphasize is that our results were obtained from scalp recordings without the use either of curare or of any filtering method. Since intracranial recordings are nearly free of artifacts, the fact that the same pattern [67] is seen in both situations reinforces the idea that the results obtained with scalp electrodes were not a spurious effect of muscle activity. Also to be noted is the fact that, although the grouping in frequency bands implies a loss of frequency resolution, this procedure can be more useful than a study of single frequencies or peaks, due to the relation between frequency bands and their "sources" in the brain. In this context, the RWE permits an easy interpretation of several minutes of frequency variations in a single display, something that is sometimes difficult to achieve with traditional scalp EEGs.

Being independent of the amplitude or the energy of the signal, the normalized wavelet entropy, $H$, yields new information about EEG signals in comparison with that obtained by using frequency analysis or other standard methods. The normalized wavelet entropy has the following advantages: 
(i) In contrast to the spectral entropy, the normalized wavelet entropy is capable of detecting changes in a non-stationary signal due to the localization characteristics of the wavelet transform. (ii) In comparison with dimensional analysis and Lyapunov exponents (which are only defined for stationary behaviors) or with dimensional and chaotic measures (stationary constraints removed), the computational time required for the evaluation of the normalized wavelet entropy is significantly shorter. The algorithm for evaluation involves just the use of the wavelet transform in a multiresolution framework. (iii) Contaminating noise contributions (if they are basically concentrated in some frequency bands) can be easily eliminated. Last but not least, (iv) no previous evaluated parameters are needed for the evaluation of normalized wavelet entropies.

One of our goals here was to introduce the notion of complexity in the wavelet scenario, so as to gather new insight into the background activity leading to (1) epileptic seizures and (2) tonic to clonic phase transitions. With the help of both the normalized wavelet entropy and the wavelet complexity notion, we detected the presence of a rather special brain-state characterized by both order and large complexity.

Another aim was to extend the methodology based on ODWT to another set of multiresolution wavelet coefficients: the wavelet leaders. It can be noted that the information quantifiers WLSS and WLSC have the same computational evaluation advantages as those based on ODWT coefficients, and they also detect changes in the EEG signal during the seizure, having a more accurate time localization and capturing the more irregular portion of the signal between some seconds before the beginning of the clonic phase and the end of the seizure. Results presented in the previous sections show that quantifiers based on extensive informational wavelet tools could be useful for the quantitative description of TCES.

The electrophysiology of generalized TCES is not fully understood. There are many descriptions of the typical patterns of EEG activity that accompany these seizures, but few detailed or quantitative analysis are given. One of the main reasons for this state of affairs is that, immediately after this kind of seizure begins, muscle activity starts leading to artifacts, which obscure the EEG data. The description of these data suggests that generalized TCES-EEG data have a complex evolution in time. Spatial variation on their manifestation has been reported, despite their classification as "generalized seizures" [63,67]. Yet, until recently, there have been no studies that have attempted to investigate and quantify these complex dynamics and there is little information to help explain the neurophysiology underlying generalized TCES. A naive assumption is to believe that there are no variations in the degree to which cortical neurons participate in the generalized TCES. However, the available data do not support such a view. Instead, the data suggest that there are variations in the degree in which cortical neurons participate in the generalized TCES in both animal models and in humans. Some researchers have even suggested that the ERR manifested in the EEG after seizure onsets is a cardinal feature of these epileptic seizures [63].

One critical point is the possible distortion due to the spatial propagation of the seizure, since data from the $\mathrm{C} 4$ electrode was analyzed and the sources of the seizures were in a temporal location. In order to overcome this difficulty, quantifiers based on ODWT were also applied to the T3 and T4 electrodes, obtaining thereby similar results to the ones reported using the $\mathrm{C} 4$ electrode. In particular, the EER was still observed, even though these electrodes exhibit more artifacts than the $\mathrm{C} 4$. In addition, the seizure stage presents smaller normalized wavelet entropy and larger wavelet SCM values in comparison to the pre-seizure one. For a quantitative analysis of the behavior observed in these two electrodes (T3 and T4), 
the above introduced informational quantifiers based on ODWT were evaluated with the same thresholds. The number of cases for which the imposed constraints for the quantifiers are satisfied is similar for C4. This implies that the method is "transportable" to different electrodes. Although some variations in the numerical values for the different quantifiers were observed, it is reasonable to assume that the different positions could provide additional information.

Figure 11. Temporal evolution of the wavelet leader Shannon entropy for the original signals corresponding to tonic-clonic epileptic EEG records in different channels. The vertical lines indicate the following transitions: The seizure starts at $\mathbf{T I}=80 \mathrm{~s}$ and the clonic phase at $\mathbf{T} 2$ $=125 \mathrm{~s}$. The seizure ends at $\mathbf{T F}=155 \mathrm{~s}$. $\mathbf{T} 1=90 \mathrm{~s}$ and $\mathbf{T} 3=145 \mathrm{~s}$ mark the expected beginning and end of the epileptic recruiting rhythm (ERR).

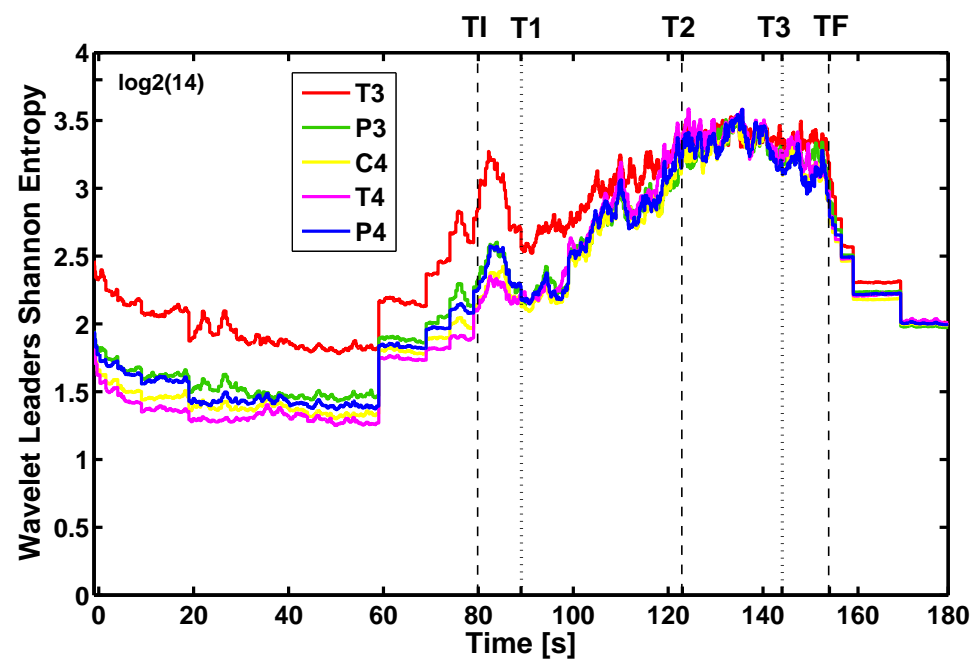

Figure 12. Temporal evolution of the wavelet leader statistical complexity for the original signals corresponding to tonic-clonic epileptic EEG records in different channels. The vertical lines indicate the following transitions: The seizure starts at TI $=80 \mathrm{~s}$ and the clonic phase at $\mathbf{T} 2=125 \mathrm{~s}$. The seizure ends at $\mathbf{T F}=155 \mathrm{~s}$. $\mathbf{T} 1=90 \mathrm{~s}$ and $\mathbf{T} 3=145 \mathrm{~s}$ mark the expected beginning and end of the epileptic recruiting rhythm (ERR).

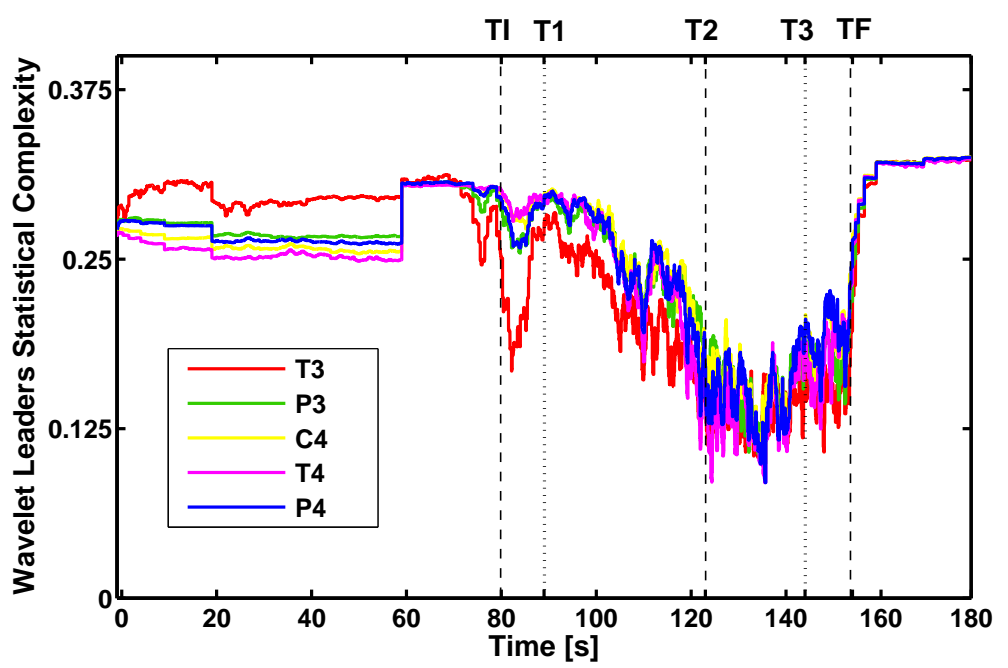


In this view, it is interesting to observe that information quantifiers based on wavelet leaders (WLSS and WLSC) are not significantly affected by including the bands related to the muscle activity, and this signal analysis for other electrodes is similar and closely associated with the analysis of the $\mathrm{C} 4$ electrode. This fact can be observed in Figures 11 and 12, which display the information quantifiers (WLSS and WLSC) corresponding to the original EEG records from electrodes at the temporal and parietal locations, T3, T4, P3, P4, respectively, as well as the previously analyzed channel, C4. We have selected these few channels, taking into account that the source of the seizure is left temporal.

\section{Final Remarks}

It is well established that an EEG is directly proportional to the local field potential recorded by electrodes on the brain surface. Furthermore, one single EEG electrode placed on the scalp records the aggregate electrical activity from up to $6 \mathrm{~cm}^{2}$ of the brain surface and, hence, from many millions of neurons. With such large numbers, it seems quite natural to model the neocortex as a continuous sheet of neurons (neuronal matter) whose activity varies with time. Taking into account the results that we have obtained for: (1) the chaoticity (biggest Lyapunov exponent with stationary constrained removed) as a function of time [7,19] and (2) the biggest Lyapunov exponent for the selected portion of the EEG signal, we conclude that a chaotic behavior can be associated with the whole EEG signal, although it becomes less noticeable during the recruiting phase $[7,19]$ ). We have shown here that the recruiting phase also exhibits larger values of statistical complexity. As pointed out by many authors (see, for instance, [20]), the coexistence of chaos with ordering and increasing complexity for an extended system is a manifestation of self-organization. On the basis of (1) experimental EEG data and (2) the use of appropriate statistical tools, the following conjecture could be proposed in the case of secondary generalized TCES: the epileptic focus triggers a self-organized brain state characterized by both order and maximal complexity. As a consequence, it becomes clear that quantifiers of the epileptic recruiting rhythm are useful tools for the characterization of the genesis and spreading of this type of seizure. In this context, we consider that the wavelet-based informational tools reviewed in the present work and their evaluation could be profitably employed for developing models, as well as for illuminating a number of aspects of the spatio-temporal dynamics of the generalized TCES-EEG data that had not previously been appreciated.

A goal of this work was to compare and examine global and pointwise information theory-based quantifiers, constructed from two different pdfs, (1) the normalized Shannon wavelet entropy (SWS) and wavelet statistical complexity (SWC), which are based on the energy of different frequency bands present in the signal, as opposed to (2) the wavelet leader Shannon entropy (WLSS) and wavelet leader statistical complexity (WLSC), which are related to the measure of local regularity at each point of the time series. Note that both use sets of multiresolution wavelet analysis coefficients.

As the first general question, we can see that both groups of quantifiers distinguish the seizure, which is no great achievement, because it can be seen with the naked eye, but quantitative analysis tools have been developed over the years, thereby introducing an objective method of measure. About the differences between orthogonal discrete wavelet coefficients and wavelet leader coefficient-based quantifiers, we can say that these last have the following advantages: 
1. The analysis is local, and wavelet leader-based quantifiers can detect a higher resolution in the changes of the signals than Shannon wavelet entropy and wavelet statistical complexity. Moreover, the video recordings of the patient present some inaccurate information on these changes.

2. As mentioned above, the results obtained for the filtered signal (i.e., without the contribution of muscle-noise contaminated bands) is essentially identical to the original signal. This property has two advantages, it reduces the use of mathematical tools, such as signal pre-processing filters, and second, it simplifies the calculation process.

3. Pre-seizure and post-seizure fluctuation values in the evaluation of SWS and WSC quantifiers make average evaluation necessary to find the plateau that occurs naturally in wavelet leader-based quantifiers (WLSS and WLSC). Moreover, this fact clearly marks discontinuities in the signal (such as shown at $\sim 60 \mathrm{~s}$ ).

4. The maximum wavelet leader Shannon entropy WLSS occurs slightly after $120 \mathrm{~s}$, marking a transition from the tonic to the clonic phase, in agreement with what was found by computing the SWS and WSC.

It is worth noting that a massive presence of epileptic spikes with the highest rugged peaks appears during the seizure. One could guess that the jumps of the wavelet leader-based quantifiers (WLSS and WLSC) shown at $60 \mathrm{~s}$ correspond to a significant variation in the spikes, indicating that a seizure is coming. It has also been exhibited that WLSS and WLSC are sharper than the pointwise Hölder exponent to differentiate the epileptic seizure from the pre-and post-seizure stages. This fact is in correspondence with the results presented in [32] for another time series, coming from a very different source.

Finally, we consider these two analysis techniques to be complementary to each other. While SWS and WSC quantifiers are based on computing the change in the frequencies of the signals, the WLSS and the WLSC consider the regularity of the signal, describing its morphological changes.

\section{Acknowledgments}

The comments of the two anonymous reviewers were responsible for a significant improvement in the manuscript; we are grateful to them. This work was partially supported by Consejo Nacional de Investigaciones Científicas y Técnicas (CONICET). M.R., G.P. and A.F. are very thankful to Universidad Nacional de General Sarmiento. E.S. wishes to thank Universidad de San Martín, and O.A.R. gratefully acknowledges support from CONICET, Argentina, and FAPEAL, Brazil.

\section{Author Contributions}

All authors contributed equally in the design of this research as well as in the writing of this paper. All authors have read and approved the final manuscript.

\section{Conflicts of Interest}

The authors declare no conflict of interest. 


\section{References}

1. Niedermeyer, E.; Lopes da Silva, F.H. Electroencephalography, Basic Principles, Clinical Applications, and Related Field; Urban and Schwarzenberg: Baltimore, MD, USA, 1987.

2. Casdagli, M.C.; Iasemedis, L.D.; Savit, R.S.; Gilmore, R.L.; Roper, S.N.; Sackellares, J.C. Non-linearity in invasive EEG recordings from patients with temporal lobe epilepsy. Electroencephalogr. Clin. Neurophysiol. 1997, 102, 98-105.

3. Lehnertz, K.; Elger, C.E. Can epileptic seizures be predicted? Evidence from nonlinear time series analysis of brain electrical activity. Phys. Rev. Lett. 1998, 80, 5019-5022.

4. Kowalik, Z.J.; Elbert, T. Changes of chaoticness in spontaneous EEG/EMG. Integr. Physiol. Behav. Sci. 1994, 29, 270-282.

5. Iasemedis, L.D.; Sackellares, J.C.; Zaveri, H.P.; Williams, W.J. Phase space topographyand Lyapunov exponent of electrocorticograms in partial seizures. Brain Topogr. 1990, 2, 187-201.

6. Iasemedis, L.D. Sackellares, J.C. The evolution with time of spatial distribution of the largest Lyapunov exponent on the human epileptic cortex. In It Measuring Chaos in Human Brain; Duke, D., Pritchards, W., Eds.; World Scientific: Singapore, Singapore, 1991; pp. 49-82.

7. Rosso, O.A.; Mairal, M.L. Characterization of time dynamical evolution of electroencephalographic epileptic records. Physica A 2002, 312, 469-504.

8. Pijn, J.P.; van Neerven, J.; Noestt, A.; Lopes da Silva, F.H. Chaos or noise in EEG signals: Dependence on state and brain site. Electroencephalogr. Clin. Neurophysiol. 1991, 79, 371-381.

9. Powell, G.E.; Percival, I.C. A spectral entropy method for distinguishing regular and irregular motion of Hamiltonian systems. J. Phys. A Math. Gen. 1979, 12, 2053-2071.

10. Daubechies, I. Ten Lectures on Wavelets; SIAM: Philadelphia, PA, USA, 1992.

11. Mallat, S. A Wavelet Tour of Signal Processing, 3rd ed.; Academic Press: Burlington, MA, USA, 2009.

12. Samar, V.; Bopardikar, A.; Rao, R.; Swartz, K. Wavelet analysis of neuroelectric waveforms: A conceptual tutorial. Brain Lang. 1999, 66, 7-60.

13. Blanco, S.; Figliola, A.; Quian Quiroga, R.; Rosso, O.A.; Serrano, E. Time-Frequency analysis of electroencephalogram series (III): Information transfer function and wavelets packets. Phys. Rev. E 1998, 57, 932-940.

14. Rosso, O.A.; Martín, M.T.; Figliola, A.; Keller, K.; Plastino, A. EEG analysis using wavelet-based informational tools. J. Neurosci. Methods 2006, 153, 163-182.

15. Rosso, O.A., Blanco, S.; Yordanova, J.; Kolev, V.; Figliola, A.; Schürmann, M.; Başar, E. Wavelet entropy: A new tool for the analysis of short duration brain electrical signals. J. Neurosci. Methods 2001, 105, 65-75.

16. Rosso, O.A.; Martín, M.T.; Plastino, A. Brain electrical activity analysis using wavelet based informational tools. Physica A 2002, 313, 587-608.

17. Rosso, O.A.; Blanco, S.; Rabinowicz, A. Wavelet analysis of generalized Tonic-Clonic epileptic seizures. Signal Process. 2002, 83, 1275-1289. 
18. Rosso, O.A.; Martín, M.T.; Plastino, A. Brain electrical activity analysis using wavelet based informational tools (II): Tsallis non-extensivity and complexity measurements. Physica A 2003, 320, 497-511.

19. Rosso, O.A.; Figliola, A. Order/Disorder in brain electrical activity. Rev. Mex. Física 2004, 50, 149-155.

20. Rosso, O.A.; Martín, M.T.; Plastino, A. Evidence of self-organization in brain electrical activity using wavelet based informational tools. Physica A 2005, 347, 444-464.

21. Rosso, O.A.; Hyslop, W.; Gerlach, R.; Smith, R.L.L.; Rostas, J.; Hunter, M. Quantitative EEG analysis of the maturational changes associated with childhood absence epilepsy. Physica $A \mathbf{2 0 0 5}$, 356, 184-189.

22. Rosso, O.A. Entropy changes in brain function. Int. J. Psychophysiol. 2007, 64, 75-80.

23. Pereyra, M.; Lamberti, P.W.; Rosso, O.A. Wavelet Jensen-Shannon divergence as a tool for studying the dynamics of frequency band components in EEG epileptic seizures. Physica A 2007, 379, $122-132$

24. Rosso, O.A., Mendes, A.; Rostas, J.A.; Hunter, M.; Moscato, P. Distinguishing childhood absence epilepsy patients from controls by the analysis of their background brain electrical activity. J. Neurosci. Methods 2009, 177, 461-468.

25. Rosso, O.A.; Mendes, A.; Berreta, R.; Rostas, J.A.; Hunter, M.; Moscato, P. Distinguishing childhood absence epilepsy patients from controls by the analysis of their background brain activity (II): A combinatorial optimization approach for electrode selection. J. Neurosci. Methods 2009, $181,257-267$.

26. Quian Quiroga, R.; Rosso, O.A.; Başar, E.; Schürmann, M. Wavelet-entropy in event-related potentials: A new method shows ordering of EEG oscillations. Biol. Cybern. 2001, 84, 291-299.

27. Kolev, V.; Rosso, O.A.; Yordanova, J. A transient dominance of theta ERP component characterizes passive auditory processing: Evidence from developmental study. Neuroreport 2001, 12, 2791-2796.

28. Yordanova, J.; Kolev, V.; Rosso, O.A.; Schürmann, M.; Sakowitz, O.W.; Özgören, M.; Başar, E. Wavelet entropy analysis of event-related potentials indicates modality-independent theta dominance. J. Neurosci. Methods 2002, 117, 99-109.

29. Schüett, A.; Rosso, O.A.; Figliola, A. A discovery of new features of gastropod local field potentials by application of wavelet tools. J. Neurosci. Methods 2002, 119, 89-104.

30. Yordanova, J.; Rosso, O.A.; Kolev, V. A transient dominance of theta event-related brain potential component characterizes stimulus processing in auditory oddball task. Clin. Neurophysiol. 2003, 114, 529-540.

31. Schüett, A.; Rosso, O.A.; Figliola, A. Wavelet analysis can sensitively describe dynamics of ethanol evoked spiky local field potential wave of slug (Limax marginatus) brain. Clin. Neurophysiol. 2003, 129, 135-150.

32. Rosenblatt, M.; Serrano, E.; Figliola, A. An entropy based in wavelet leaders to quantify the local regularity of a signal and its application to analyze the Dow Jones index. Int. J. Wavelets Multiresolution Inf. Process. 2012, 10, 1250048. 
33. Jaffard, S. Oscillation spaces: Properties and applications to fractal and multifractal functions. J. Math. Phys. 1998 , 39, 4129-4141.

34. Jaffard, S. Wavelet techniques in multifractal analysis. Proc. Symp. Pure Math. Am. Math. Soc. 2004, 72, 91-151.

35. Arneodo, A.; Bacry, E; Muzy, J.F. The thermodynamics of fractals revisited with wavelets. Physica A 1995, 213, 232-275.

36. Lashermes, B.; Roux, S.G.; Abry, P.; Jaffard, S. Comprehensive multifractal analysis of turbulent velocity using the wavelet leaders. Eur. Phys. J. B 2008, 61, 201-215.

37. Wendt, H; Roux, S.G.; Abry, P.; Jaffard, S. Wavelet leaders and bootstrap for multifractal analysis of images. Signal Process. 2009, 89, 1100-1114.

38. Leonarduzzi, R.F.; Schlotthauer, G.; Torres, M.E. Wavelet leader based multifractal analysis of heart rate variability during myocardial ischaemia. In Proceedings of the 2010 Annual International Conference of the IEEE Engineering in Medicine and Biology Society (EMBC), Buenos Aires, Argentina, 31 August-4 Septermber 2010; pp. 110-113.

39. Abry, P.; Roux S.G.; Jaffard, S. Detecting oscillating singularities in multifractal analysis: Application to hydrodynamic turbulence. In Proceedings of the IEEE International Conference On Acoustics, Speech, and Signal Processing, Prague, Czech Republic, 22-27 May 2011.

40. Mallat, S. Multiresolution approximations and wavelet orthonormal bases of $L^{2}$. Trans. Am. Math. Soc. 1989, 315, 69-87.

41. Meyer, Y. Ondelettes et Opérateurs; Hermann: Paris, France, 1990.

42. Unser, M. Splines a perfect fit for signal and image processing. IEEE Signal Process. Mag. 1999, 16, 22-38.

43. Thevenaz P.; Blue T.; Unser, M. Interpolation revisited. IEEE Trans. Med. Imaging 2000, 19, 739-758.

44. Shannon, C.E. A mathematical theory of communication. Bell Syst. Technol. J. 1948, 27, 379-423 and 623-656.

45. Cover, T.M.; Thomas, J.A. Elements of Information Theory; Wiley: New York, NY, USA, 1991.

46. Kolmogorov, A.N. A new metric invariant of transitive dynamic system and automorphysms in Lebesgue spaces. Dokl. Akad. Nauk. SSSR 1958, 119, 861-864.

47. Sinai, Y.G. On the concept of entropy of dynamical system. Dokl. Akad. Nauk. SSSR 1959, 124, 768-771.

48. Feldman, D.P.; Crutchfield, J.P. Measures of statistical complexity: Why? Phys. Lett. A 1998, 238, 244-252.

49. López-Ruiz, R.; Mancini, H.; Calbet, X. A statistical measure of complexity. Phys. Lett. A 1995, 209, 321-326.

50. Martín, M.T.; Plastino, A.; Rosso, O.A. Statistical complexity and disequilibrium. Phys. Lett. A 2003, 311, 126-132.

51. Lamberti, P.W.; Martín, M.T.; Plastino, A.; Rosso, O.A. Intensive entropic nontriviality measure. Physica A 2004, 334, 119-131.

52. Grosse, I.; Bernaola-Galván, P.; Carpena, P.; Román-Roldán, R.; Oliver, J.; Stanley, H.E. Analysis of symbolic sequences using the Jensen-Shannon divergence. Phys. Rev. E 2002, 65, 041905. 
53. Martín, M.T.; Plastino, A.; Rosso, O.A. Generalized statistical complexity measures: Geometrical and analytical properties. Physica A 2006, 369, 439-462.

54. Loṕez-Ruiz, R.; Sañudo, J.; Romera, E.; Calbet, X. Statistical Complexity and Fisher-Shannon Information: Applications; Springer Netherlands: Berlin, Germany, 2012.

55. Legrand, P. Local regularity and multifractal methods for image and signal analysis. In Scaling, Fractals and Wavelets; Abry, P., Gonçalvès, P., Lévy Vehel, J., Eds.; Iste and Wiley: London, UK, 2009; pp. 367-409.

56. Lévy Véhel, J.; Seuret, S. Two-microlocal formalism. Fractal Geom. Appl. Jubil. Benoit Mandelbrot Proc. Symp. Pure Math. 2004, 72, 153-215.

57. Meyer, Y. Wavelets, Vibrations and Scalings; American Mathematical Society - CRM: Providence, RI, USA, 1997.

58. Jaffard, S. Exposants de Hölder en des points donnés et coefficients d'ondelettes. C. R. Acad. Sci. Ser. I Math. 1989, 308, 79-81.

59. Gevins, A., Rémond, R., Eds. Handbook of Electroencephalography and Clinical Neurophysiology, Vol. I: Methods of Analysis of Brain Electrical and Magnetic Signals; Elsevier: Amsterdam, The Netherlands, 1987.

60. Lopes da Silva, F.H.; van Leeuwen, S.; Rémond, A. Handbook of Electroencephalography and Clinical Neurophysiology, Vol. II: Clinical Applications of Computer Analysis of EEG and other Neurophysiological Signals; Elsevier: Amsterdam, The Netherlands, 1986.

61. Daly, D.D., Pedley, T.A., Eds. Current Practice of Clinical Electroencephalography, 2nd ed.; Lippincott Williams \& Wilkins: Philadelphia, PA, USA, 1990.

62. Gastaut, H.; Fischer-Williams, M. In Handbook of Physiology; Field, J., Magoun, H.W., Hall, V.E., Eds.; Williams \& Wilkins: Baltimore, MD, USA, 1959; Volume 1.

63. Gastaut, H.; Broughton, R. Epileptic Seizures; Thomas: Springfield, IL, USA, 1972.

64. Gotman, J.; Ives, J.R.; Gloor, P. Frequency content of EEG and EMG at seizure onset: Possibility of removal of EMG artifact by digital filtering. Clin. Neurophysiol. 1981, 52, 626-639.

65. Shang, P.; Lu, Y.; Kama, S. The application of Hölder exponent to traffic congestion warning. Physica A 2006, 370, 769-776.

66. Quiroga, R.Q. Quantitative Analysis of EEG Signals: Time-Frequency Methods and Chaos Theory. Ph.D. Thesis, Institute of Physiology and Institute of Signal Processing, Medical University of Lübeck, Lübeck, Germany, 1998.

67. Darcey, T.M.; Willamson, P.D. Spatio-temporal EEG measures and their application to human intracranialy recorded epileptic seizures. Electroencephalogr. Clin. Neurophysiol. 1985, 61, 573-587.

(c) 2014 by the authors; licensee MDPI, Basel, Switzerland. This article is an open access article distributed under the terms and conditions of the Creative Commons Attribution license (http://creativecommons.org/licenses/by/4.0/). 